This item was submitted to Loughborough's Research Repository by the author.

Items in Figshare are protected by copyright, with all rights reserved, unless otherwise indicated.

\title{
Two-parameter p, q-variation paths and integrations of local times
}

PLEASE CITE THE PUBLISHED VERSION

LICENCE

CC BY-NC-ND 4.0

\section{REPOSITORY RECORD}

Feng, Chunrong, and Huaizhong Zhao. 2019. "Two-parameter P, Q-variation Paths and Integrations of Local Times". figshare. https://hdl.handle.net/2134/1279. 


\title{
Two-parameter $p, q$-variation Paths and Integrations of Local Times
}

\author{
Chunrong Feng ${ }^{1,2}$, Huaizhong Zhao ${ }^{1}$ \\ 1 Department of Mathematical Sciences, Loughborough University, LE11 3TU, \\ UK. C.Feng@lboro.ac.uk, H.Zhao@lboro.ac.uk \\ 2 School of Mathematics and System Sciences, Shandong University, Jinan, \\ Shandong Province, 250100, China
}

Summary. In this paper, we prove two main results. The first one is to give a new condition for the existence of two-parameter $p, q$-variation path integrals. Our condition of locally bounded $p, q$-variation is more natural and easy to verify than those of Young. This result can be easily generalized to multi-parameter case. The second result is to define the integral of local time $\int_{-\infty}^{\infty} \int_{0}^{t} g(s, x) d_{s, x} L_{s}(x)$ pathwise and then give generalized Itô's formula when $\nabla^{-} f(s, x)$ is only of bounded $p, q$-variation in $(s, x)$. In the case that $g(s, x)=\nabla^{-} f(s, x)$ is of locally bounded variation in $(s, x)$, the integral $\int_{-\infty}^{\infty} \int_{0}^{t} \nabla^{-} f(s, x) d_{s, x} L_{s}(x)$ is the Lebesgue-Stieltjes integral and was used in Elworthy, Truman and Zhao (2004). When $g(s, x)=\nabla^{-} f(s, x)$ is of only locally $p, q$-variation, where $p \geq 1, q \geq 1$, and $2 q+1>2 p q$, the integral is a twoparameter Young integral of $p, q$-variation rather than a Lebesgue-Stieltjes integral. In the special case that $f(s, x)=f(x)$ is independent of $s$, we give a new condition for Meyer's formula and $\int_{-\infty}^{\infty} L_{t}(x) d_{x} \nabla^{-} f(x)$ is defined pathwise as a Young integral. For this we prove the local time $L_{t}(x)$ is of $p$-variation in $x$ for each $t \geq 0$, for each $p>2$ almost surely ( $p$-variation in the sense of Lyons and Young, i.e. $\left.\sup _{E: \text { a finite partition of }[-N, N]} \sum_{i=1}^{m}\left|L_{t}\left(x_{i}\right)-L_{t}\left(x_{i-1}\right)\right|^{p}<\infty\right)$.

Keywords: Two-parameter $p, q$-variation path integral, local time, continuous semimartingale, generalized Itô's formula.

\section{Introduction}

The classical Itô's formula for twice differentiable functions has played a central role in stochastic analysis and almost all aspects of its applications and connection with analysis, PDEs, geometry, dynamical systems, finance and physics. But the restriction of Itô's formula to functions with twice differentiability often encounter difficulties in applications. Extensions to less smooth functions are useful in studying many problems such as partial differential equtions with some singularities and mathematics of finance. Generally speaking, for any absolutely continuous function whose derivative $f^{\prime}$ exists almost everywhere, and a continuous semi-martingale $X_{t}$, there exists $A_{t}$ such that 


$$
f\left(X_{t}\right)=f\left(X_{0}\right)+\int_{0}^{t} f^{\prime}\left(X_{s}\right) d X_{s}+A_{t}
$$

and for the time dependent case, the corresponding formula is

$$
f\left(t, X_{t}\right)=f\left(0, X_{0}\right)+\int_{0}^{t} \frac{\partial}{\partial s} f\left(s, X_{s}\right) d s+\int_{0}^{t} \nabla f\left(s, X_{s}\right) d X_{s}+A_{t} .
$$

To find $A_{t}$ in both cases especially a pathwise formula becomes key to establish a useful extension to Itô's formula. In fact investigations already began in Tanaka [28] with a beautiful use of local times introduced in Lévy [17]. The generalized Itô's formula in one-dimension for time independent convex functions was developed in Meyer [22] and for superharmonic functions in multidimensions in Brosamler [5] and for distance function in Kendall [15] and more recently for time dependent functions in Peskir [25] and Elworthy, Truman and Zhao [6]. Meyer [22] proved if $f$ is a convex function (or difference of two convex functions), then

$$
f\left(X_{t}\right)=f\left(X_{0}\right)+\int_{0}^{t} \nabla^{-} f\left(X_{s}\right) d X_{s}+\int_{-\infty}^{\infty} L_{t}(x) d_{x} \nabla^{-} f(x) \text { a.s. }
$$

where $\nabla^{-} f(x)$ is of bounded variation and $\int_{-\infty}^{\infty} L_{t}(x) d_{x} \nabla^{-} f(x)$ is a LebesgueStieltjes integral associated with the measure $d_{x} \nabla^{-} f(x)$. Elworthy, Truman and Zhao [6] proved if $f(t, x)=f_{h}(t, x)+f_{v}(t, x)$, where $\Delta^{-} f_{h}(t, x)$ and $\nabla^{-} f(t, x)$ exist and are left continuous, and $\nabla^{-} f_{v}(t, x)$ is of locally bounded variation in $x$ for a fixed $t$ and of locally bounded variation in $(t, x)$, then

$$
\begin{aligned}
& f(t, X(t))-f(0, X(0)) \\
= & \int_{0}^{t} \frac{\partial^{-}}{\partial s} f(s, X(s)) \mathrm{d} s+\int_{0}^{t} \nabla^{-} f(s, X(s)) d X_{s} \\
& +\frac{1}{2} \int_{0}^{t} \Delta^{-} f_{h}(s, X(s)) d<X>_{s}+\int_{-\infty}^{\infty} L_{t}(x) \mathrm{d}_{x} \nabla^{-} f_{v}(t, x) \\
& -\int_{-\infty}^{+\infty} \int_{0}^{t} L_{s}(x) \mathrm{d}_{s, x} \nabla^{-} f_{v}(s, x) \quad \text { a.s. }
\end{aligned}
$$

where $\int_{-\infty}^{+\infty} \int_{0}^{t} L_{s}(x) \mathrm{d}_{s, x} \nabla^{-} f_{v}(s, x)$ is a space-time Lebesgue-Stieltjes integral and needless to say, defined pathwise. Elworthy-Truman-Zhao's formula was given in a very general form. It includes as special cases classical Itô's formula, Tanaka's formula, Meyer's formula, Azema-Jeulin-Knight-Yor's formula [2]. A special and earlier version of Elworthy-Truman-Zhao's formula was obtained by Peskir [24] independently. Feng and Zhao [9] extended (1.4) to two dimensions. Noticing that the nonexistence of local time in two dimensions gives an essential difficulty in extending (1.4) to 2-dimensions, so the extension was nontrivial and the key was to define the stochastic Lebesgue-Stieltjes integral. 
On the other hand, there are some works to define $\int_{-\infty}^{\infty} \nabla^{-} f(x) d_{x} L_{t}(x)$ and $\int_{-\infty}^{\infty} \int_{0}^{t} \nabla^{-} f(s, x) d_{s, x} L_{s}(x)$ in $L^{2}(d P)$ or in $L^{1}(d P)$ in connection with Itô's formula by using terms in (1.3) other than the last term or backwardforward stochastic integrals (Bouleau and Yor [4], Eisenbaum [7], [8], Flandoli, Russo, Wolf [10], Föllmer and Protter [11], Moret and Nualart [23], etc.) and the work of Rogers and Walsh [27] using excursion fields. Generally speaking, one expects stronger conditions for the pathwise existence of the integrals of local times. However, in the framework of Lebesgue integrals, locally bounded variation in $x$ for fixed $t$ and locally bounded variation in $(t, x)$ are minimal conditions on $\nabla^{-} f(t, x)$ to generate a measure, so it seems impossible to go beyond Elworthy-Truman-Zhao's formula. We remark that the striking fact that $L_{t}(x)$ is of bounded quadratic variation in $x$ in the sense of Revuz and Yor [26] and increasing in $t$ did not play a significant role in the proof of (1.4). It is therefore reasonable to conjecture that the conditions in [6] defining the integrals of local times pathwise can be weakened. Inevitably, we have to go beyond Lebesgue integral as it seems to us that Elworthy-Truman-Zhao's formula has achieved the best in the Lebesgue integral framework. Here we use Young's idea of integration (Lyons [18], [19], Lyons and Qian [20], Young [30], [31]) to define the integral of local time to go beyond the bounded variation condition. We would like to remark that the quadratic variation in the sense of Revuz and Yor is not enough to define Young integral for local times. So it is necessary to prove local time $L_{t}(x)$ is of bounded $p$-variation for each $p>2$ in the sense of Young almost surely. The main difficulty is overcome by using the idea of controlling the $p$-variation of continuous paths via the variations through dyadic partitions. This idea was originated by Lévy, used in [3], [13], [16] to prove the Brownian path is of bounded variation for $p>2$.

Using Young's integration of one parameter $p$-variation, we can immediately define $\int_{-\infty}^{\infty} \nabla^{-} f(x) d_{x} L_{t}(x)$ as a Young integral if $\nabla^{-} f(x)$ is of bounded $q$-variation $(1 \leq q<2)$. Then a new extension of Meyer's formula to $f$ where $\nabla^{-} f(x)$ is of bounded $q$-variation $(1 \leq q<2)$ follows immediately. However one can immediately realize the difficulty of defining the two-parameter integral when we work on time dependent $f$. Young [31] considered this problem, but his conditions are strong and difficult to check. It seems to us that the theory of two-parameter $\Phi_{1}, \Psi_{1}$-variation ( $p, q$-variation as a special case) integration has not been investigated and developed well in the literature. Inspired by the work of Young [31] and Lyons and Qian [20], in this paper, we give a new condition for the existence of two-parameter Young integral (Theorem 3.1). We consider a continuous function $F(x, y)$ being of bounded $\Phi$-variation in $x$ uniformly in $y$, and being of bounded $\Psi$ - variation in $y$ uniformly in $x ; G(x, y)$ being of bounded $\Phi_{1}, \Psi_{1}$-variation in $(x, y)$, i.e.

$$
\sup _{E \times E^{\prime}} \sum_{j=1}^{N^{\prime}} \Psi_{1}\left(\sum_{i=1}^{N} \Phi_{1}\left(\left|\Delta_{i} \Delta_{j} G\right|\right)\right)<\infty,
$$


where $E \times E^{\prime}:=\left\{x^{\prime}=x_{0}<x_{1}<\cdots<x_{N}=x^{\prime \prime}, y^{\prime}=y_{0}<y_{1}<\right.$ $\left.\cdots<y_{N^{\prime}}=y^{\prime \prime}\right\}$ is an arbitrary partition of $\left[x^{\prime}, x^{\prime \prime}\right] \times\left[y^{\prime}, y^{\prime \prime}\right],\left|\Delta_{i} \Delta_{j} G\right|=$ $\left|G\left(x_{i}, y_{j}\right)-G\left(x_{i-1}, y_{j}\right)-G\left(x_{i}, y_{j-1}\right)+G\left(x_{i-1}, y_{j-1}\right)\right|$, and $\Psi_{1}, \Phi_{1}$ are convex functions. Then if there exist monotone increasing functions $\varrho$ and $\sigma$ subject to $\varrho(u) \sigma(u)=u$ such that

$$
\sum_{m, n} \varrho\left[\varphi\left(\frac{1}{n}\right)\right] \sigma\left[\psi\left(\frac{1}{m}\right)\right] \varphi_{1}\left[\frac{1}{n} \psi_{1}\left(\frac{1}{m}\right)\right]<\infty,
$$

then the integral

$$
\begin{gathered}
\int_{x^{\prime}}^{x^{\prime \prime}} \int_{y^{\prime}}^{y^{\prime \prime}} F(x, y) d_{x, y} G(x, y) \\
=\lim _{m\left(E \times E^{\prime}\right) \rightarrow 0} \sum_{i=1}^{N} \sum_{j=1}^{N^{\prime}} F\left(x_{i-1}, y_{j-1}\right)\left(G\left(x_{i}, y_{j}\right)-G\left(x_{i-1}, y_{j}\right)\right. \\
\left.\quad-G\left(x_{i}, y_{j-1}\right)+G\left(x_{i-1}, y_{j-1}\right)\right)
\end{gathered}
$$

is well defined. Here $\phi, \psi, \phi_{1}, \psi_{1}$ are inverse functions of $\Phi, \Psi, \Phi_{1}, \Psi_{1}$, respectively. For this we use Lyons' idea of control function to two-parameter case. We also prove a dominated convergence theorem (Theorem 3.2) for the integral. Then we apply this to establish the integral of local time $\int_{-\infty}^{\infty} \int_{0}^{t} L_{s}(x) d_{s, x} \nabla^{-} f(s, x)$ pathwise, where $\nabla^{-} f(t, x)$ is of locally bounded p,q-variation with $p, q \geq 1,2 q+1>2 p q$. This is new in the literature. Under this condition we establish generalized Itô's formula with the help of the dominated convergence theorem. We believe our results of the two-parameter $p, q$-variation path integration are new and has independent interest.

To compare our condition (1.5) with that of Young, we quote his condition here

$$
\left|\Delta_{i} \Delta_{j} G\right| \leq \lambda\left(\Delta_{i} \omega\right) \mu\left(\Delta_{j} \chi\right)
$$

where $\lambda, \mu, \omega$ and $\chi$ are monotone increasing functions, and $\Delta_{i} \omega=\omega\left(x_{i}\right)-$ $\omega\left(x_{i-1}\right), \Delta_{j} \chi=\chi\left(y_{j}\right)-\chi\left(y_{j-1}\right)$. There are many examples that the condition (1.5) can be checked, e.g. $f(x, y)=x y \sin \left(\frac{1}{x}+\frac{1}{y}\right)$, for $\Phi_{1}(u)=u^{p}, \Psi_{1}(u)=$ $u$, where $p>1$. But it seems difficult, if not impossible, to check Young's condition (1.8) for this example. Needless to say, in the one-parameter case, it is easy to see that the well-known example of unbounded variation function $f(x)=x \sin \frac{1}{x}$ is of bounded $p$-variation, for any $p>1$. We can prove multiparameter Brownian sheet introduced by Walsh [29] in studying stochastic partial differential equations satisfies definition of $p, 1$-variation path $(p>2)$, therefore we can define integral w.r.t. Brownian sheet pathwise and apply this idea to study stochastic PDEs. We will publish these results in future publications.

We should point out that in this paper we only study the two-parameter integration of $p, q$-variation path. This is enough for the purpose of this paper. 
In this paper, we don't include the multiplicative integrations as Lyons [18], [19], Lyons and Qian [20] investigated for the one-parameter case. We will study this important problem in future work.

\section{One parameter integral of local time}

First we recall the definition of $p$-variation path and its integration theory (see e.g. Young [30], Lyons and Qian [20]).

Definition 2.1 We call a function $f:\left[x^{\prime}, x^{\prime \prime}\right] \rightarrow R$ is of bounded p-variation if

$$
\sup _{E} \sum_{i=1}^{m}\left|f\left(x_{i}\right)-f\left(x_{i-1}\right)\right|^{p}<\infty
$$

where $E:=\left\{x^{\prime}=x_{0}<x_{1}<\cdots<x_{m}=x^{\prime \prime}\right\}$ is an arbitrary partition of $\left[x^{\prime}, x^{\prime \prime}\right]$. Here $p \geq 1$ is a fixed real number.

From Young [30], the integral $\int_{x^{\prime}}^{x^{\prime \prime}} f(x) d g(x)=\lim _{m(E) \rightarrow 0} \sum_{i=1}^{m} f\left(\xi_{i}\right)\left(g\left(x_{i}\right)-\right.$ $\left.g\left(x_{i-1}\right)\right)$ is well defined if $f$ is of bounded $p$-variation, $g$ is of bounded $q$ variation, and $f$ and $g$ have no common discontinuities. Here $\xi_{i} \in\left[x_{i-1}, x_{i}\right]$, $p, q \geq 1, \frac{1}{p}+\frac{1}{q}>1, m(E)=\sup _{1 \leq i \leq m}\left(x_{i}-x_{i-1}\right)$.

Consider a continuous semimartingale $X_{t}$ on a probability space $(\Omega, \mathcal{F}, P)$ with the decomposition

$$
X_{t}=M_{t}+V_{t}
$$

where $M_{t}$ is a local martingale, $V_{t}$ is an adapted process of bounded variation. Then there exists semimartingale local time $L_{t}^{x}$ of $X_{t}$ as a nonnegative random field $L=\left\{L_{t}^{x}:(t, x) \in[0, \infty) \times R, \omega \in \Omega\right\}$. Note there is a different definition of variation established in Revuz and Yor [26] (see also Marcus and Rosen [21]) and the following result is known (P221, Theorem 1.21, [26]): Let $\left(\Delta_{n}\right)$ be a sequence of subdivisions of $[a, b]$ such that $\left|\Delta_{n}\right| \rightarrow 0$ as $n \rightarrow \infty$, for any nonnegative and finite random variable $S$,

$$
\lim _{n \rightarrow \infty} \sum_{\Delta_{n}}\left(L_{S}^{a_{i+1}}-L_{s}^{a_{i}}\right)^{2}=4 \int_{a}^{b} L_{S}^{x} d x+\sum_{a<x \leq b}\left(L_{S}^{x}-L_{S}^{x-}\right)^{2}<\infty,
$$

in probability. However this variation is not enough to enable us to apply Young's construction of integrals. We need the following new result to establish integrations of local times. 
Lemma 2.1 Semimartingale local time $L_{t}^{x}$ is of bounded $p$-variation in $x$ for any $t \geq 0$, for any $p>2$, almost surely.

Proof: By the usual localization argument, we may first assume that there is a constant $K$ for which $\int_{0}^{t}\left|d V_{s}\right|,\langle M, M\rangle_{t} \leq K$. By Tanaka's formula

$$
L_{t}^{x}=\left(X_{t}-x\right)^{+}-\left(X_{0}-x\right)^{+}-\widehat{M}_{t}^{x}-\widehat{V}_{t}^{x},
$$

where,

$$
\widehat{M}_{t}^{x}=\int_{0}^{t} 1_{\left\{X_{s}>x\right\}} d M_{s}, \widehat{V}_{t}^{x}=\int_{0}^{t} 1_{\left\{X_{s}>x\right\}} d V_{s} .
$$

First note the function $\varphi_{t}(x):=\left(X_{t}-x\right)^{+}-\left(X_{0}-x\right)^{+}$is Lipschitz continuous in $x$ with Lipschitz constant 2, which implies for any $p>2$ and $a_{i}<a_{i+1}$

$$
\left|\widehat{\varphi}_{t}\left(a_{i+1}\right)-\widehat{\varphi}_{t}\left(a_{i}\right)\right|^{p} \leq 2^{p}\left(a_{i+1}-a_{i}\right)^{p} .
$$

Secondly, by Hölder inequality, as $V$ is of bounded variation, so

$$
\begin{aligned}
& \left|\widehat{V}_{t}^{a_{i+1}}-\widehat{V}_{t}^{a_{i}}\right|^{p} \\
\leq & \left.\left|\int_{0}^{t} 1_{\left\{a_{i}<X_{s} \leq a_{i+1}\right\}}\right| d V_{s}\right|^{p} \\
\leq & c \int_{0}^{t} 1_{\left\{a_{i}<X_{s} \leq a_{i+1}\right\}}\left|d V_{s}\right|,
\end{aligned}
$$

where $c$ is a generic constant. To treat $\widehat{M}_{t}^{a}$, we use the method in the proof of Lemma 3.7.5 in Karatzas and Shreve [14] or Theorem 6.1.7 in Revuz and Yor [26],

$$
\begin{aligned}
& E\left|\widehat{M}_{t}^{a_{i+1}}-\widehat{M}_{t}^{a_{i}}\right|^{p} \\
= & E\left|\int_{0}^{t} 1_{\left\{a_{i}<X_{s} \leq a_{i+1}\right\}} d M_{s}\right|^{p} \\
\leq & c E\left(\int_{0}^{t} 1_{\left\{a_{i}<X_{s} \leq a_{i+1}\right\}} d<M, M>_{s}\right)^{\frac{p}{2}} \\
= & c E\left(\int_{a_{i}}^{a_{i+1}} L_{t}^{x} d x\right)^{\frac{p}{2}} \\
= & c\left(a_{i+1}-a_{i}\right)^{\frac{p}{2}} E\left(\frac{1}{a_{i+1}-a_{i}} \int_{a_{i}}^{a_{i+1}} L_{t}^{x} d x\right)^{\frac{p}{2}} \\
\leq & c\left(a_{i+1}-a_{i}\right)^{\frac{p}{2}} E \frac{1}{a_{i+1}-a_{i}} \int_{a_{i}}^{a_{i+1}}\left(L_{t}^{x}\right)^{\frac{p}{2}} d x \\
\leq & c\left(a_{i+1}-a_{i}\right)^{\frac{p}{2}} \sup _{x} E\left(L_{t}^{x}\right)^{\frac{p}{2}} .
\end{aligned}
$$


Here we used Burkholder-Davis-Gundy inequality, the occupation times formula, Jensen inequality and Fubini theorem. Now from (2.4) and using Burkholder-Davis-Gundy inequality again, we have

$$
\begin{aligned}
E\left(L_{t}^{x}\right)^{\frac{p}{2}} & \leq c E\left[\left(X_{t}-X_{0}\right)^{\frac{p}{2}}+\left(\int_{0}^{t}\left|d V_{s}\right|\right)^{\frac{p}{2}}+<M, M>_{t}^{\frac{p}{4}}\right] \\
& \leq c E<M, M>_{t}^{\frac{p}{4}}+c E\left(\int_{0}^{t}\left|d V_{s}\right|\right)^{\frac{p}{2}}+c E<M, M>_{t}^{\frac{p}{4}}<c_{1}(K, p) .
\end{aligned}
$$

Therefore it follows that

$$
E\left|\widehat{M}_{t}^{a_{i+1}}-\widehat{M}_{t}^{a_{i}}\right|^{p} \leq c\left(a_{i+1}-a_{i}\right)^{\frac{p}{2}} .
$$

Here $c$ is a constant depending on $K, p$. Now we use Proposition 4.1.1 in [20] $(i=1, \gamma>p-1)$, for any partition $\left\{a_{l}\right\}$ of $[a, b]$

$$
\sup _{D} \sum_{l}\left|\widehat{M}_{t}^{a_{l+1}}-\widehat{M}_{t}^{a_{l}}\right|^{p} \leq c(p, \gamma) \sum_{n=1}^{\infty} n^{\gamma} \sum_{k=1}^{2^{n}}\left|\widehat{M}_{t}^{a_{k}^{n}}-\widehat{M}_{t}^{a_{k-1}^{n}}\right|^{p} .
$$

The crucial thing is that the right hand side does not depend on partition $\mathrm{D}$, where

$$
a_{k}^{n}=a+\frac{k}{2^{n}}(b-a), k=0,1, \cdots, 2^{n} .
$$

We take expectation

$$
\begin{aligned}
& E \sum_{n=1}^{\infty} n^{\gamma} \sum_{k=1}^{2^{n}}\left|\widehat{M}_{t}^{a_{k}^{n}}-\widehat{M}_{t}^{a_{k-1}^{n}}\right|^{p} \\
= & \sum_{n=1}^{\infty} n^{\gamma} \sum_{k=1}^{2^{n}} E\left|\widehat{M}_{t}^{a_{k}^{n}}-\widehat{M}_{t}^{a_{k-1}^{n}}\right|^{p} \\
\leq & c \sum_{n=1}^{\infty} n^{\gamma}\left(\frac{b-a}{2^{n}}\right)^{\frac{p}{2}-1}<\infty \text { as } p>2 .
\end{aligned}
$$

Therefore

$$
\sum_{n=1}^{\infty} n^{\gamma} \sum_{k=1}^{2^{n}}\left|\widehat{M}_{t}^{a_{k}^{n}}-\widehat{M}_{t}^{a_{k-1}^{n}}\right|^{p}<\infty \text { a.s.. }
$$

It turns out that for any interval $[a, b] \subset R$

$$
\sup _{D} \sum_{l}\left|\widehat{M}_{t}^{a_{l+1}}-\widehat{M}_{t}^{a_{l}}\right|^{p}<\infty \text { a.s.. }
$$

But we know for each $\omega, L_{t}(a)$ has a compact support in $a$, say $[-N, N]$ contains its support. So for the partition $D:=D_{-N, N}=\left\{-N=x_{0}<x_{1}<\right.$ $\left.\cdots<x_{r}=N\right\}$, we obtain 


$$
\sup _{D} \sum_{i}\left|\widehat{M}_{t}^{a_{i+1}}-\widehat{M}_{t}^{a_{i}}\right|^{p}<\infty \text { a.s.. }
$$

On the other hand, it is easy to see from (2.5) that

$$
\begin{aligned}
\sum_{i}\left|\varphi_{t}\left(a_{i+1}\right)-\varphi_{t}\left(a_{i}\right)\right|^{p} & \leq 2^{p} \sum_{i}\left(a_{i+1}-a_{i}\right)^{p} \\
& \leq 2^{p}\left[\sum_{i}\left(a_{i+1}-a_{i}\right)\right]^{p}=2^{p}(b-a)^{p},
\end{aligned}
$$

and from (2.6) and bounded variation of $V$ that

$$
\sum_{i}\left|\widehat{V}_{t}^{a_{i+1}}-\widehat{V}_{t}^{a_{i}}\right|^{p} \leq c \int_{0}^{t} 1_{\left\{a<X_{s} \leq b\right\}}\left|d V_{s}\right| \leq c \int_{0}^{t}\left|d V_{s}\right|<\infty .
$$

Then from (2.4), (2.8), (2.9), (2.10), we know that

$$
\sup _{D} \sum_{i}\left|L_{t}^{a_{i+1}}-L_{t}^{a_{i}}\right|^{p}<\infty \quad \text { a.s.. }
$$

Finally we can use the usual localization procedure to remove the assumption that $\int_{0}^{t}\left|d V_{s}\right|,<M, M>_{t} \leq K$. For this, define a stopping time for an integer $K>0: \tau_{K}=\inf \left\{s: \min \left\{\int_{0}^{s}\left|d V_{r}\right|,<M, M>_{s}\right\}>K\right\}$ if there exists $s$ such that $\min \left\{\int_{0}^{s}\left|d V_{r}\right|,\left\langle M, M>_{s}\right\}>K\right.$ and $\tau_{K}=+\infty$ otherwise. Then the above result shows that there exists $\Omega_{1} \subset \Omega$ with $P\left(\Omega_{1}\right)=1$ such that for each $\omega \in \Omega_{1}$ and each given integer $K>0$,

$$
\sup _{D} \sum_{i}\left|L_{t \wedge \tau_{K}}^{a_{i+1}}-L_{t \wedge \tau_{K}}^{a_{i}}\right|^{p}<\infty
$$

Since $\int_{0}^{t}\left|d V_{s}\right|(\omega)$ and $\left\langle M, M>_{t}\right.$ are finite almost surely so there exists $\Omega_{2} \subset \Omega$ with $P\left(\Omega_{2}\right)=1$ such that for each $\omega \in \Omega_{2}$, there exists an integer $K(\omega)>0$ such that $\int_{0}^{t}\left|d V_{s}\right|(\omega),\left\langle M, M>_{t}(\omega) \leq K\right.$. This leads to $\tau_{K}(\omega)>$ $t$. So for each $\omega \in \Omega_{1} \cap \Omega_{2}$,

$$
\sup _{D} \sum_{i}\left|L_{t}^{a_{i+1}}-L_{t}^{a_{i}}\right|^{p}<\infty .
$$

The result follows as $P\left(\Omega_{1} \cap \Omega_{2}\right)=1$.

$\diamond$

Recall the well-known result (see Revuz and Yor [26], P220) that for each $t$, the random function $x \rightarrow L_{t}^{x}$ is a cadlag function hence only admits at most countably many discontinuous points. Denote $\widehat{L}_{t}^{x}=L_{t}^{x}-L_{t}^{x-}$. Then

$$
\widehat{L}_{t}^{x}=\int_{0}^{t} 1_{\{x\}}\left(X_{s}\right) d V_{s}
$$


and for any $a<b$,

$$
\sum_{a<x \leq b}\left|\widehat{L}_{t}^{x}\right|=\int_{0}^{t}\left|d V_{s}\right|<\infty
$$

Write

$$
L_{t}^{x}=\tilde{L}_{t}^{x}+\sum_{x_{k}^{*} \leq x} \widehat{L}_{t}^{x_{k}^{*}}
$$

Here $\tilde{L}_{t}^{x}$ is continuous in $x$, and $\left\{x_{k}^{*}\right\}$ are the discontinuous points of $L_{t}^{x}$. Denote

$$
h(t, x):=\sum_{x_{k}^{*} \leq x} \widehat{L}_{t}^{x_{k}^{*}}
$$

Lemma 2.2 Above defined $h(t, x)$ is of bounded variation in $x$ for each $t$ and of bounded variation in $(t, x)$ for almost every $\omega \in \Omega$.

Proof: Let $[-N, N]$ be the support of $L_{t}(x)$. To see that $h(t, x)$ is of locally bounded variation in $x$, consider any partition $D=\left\{-N=x_{0}<x_{1}<\cdots<\right.$ $\left.x_{m-1}<x_{m}=N\right\}$, then from $(2.12)$

$$
\begin{aligned}
\sum_{i}\left|h\left(t, x_{i+1}\right)-h\left(t, x_{i}\right)\right| & =\sum_{i}\left|\sum_{x_{i}<x_{k}^{*} \leq x_{i+1}} \widehat{L}_{t}^{x_{k}^{*}}\right| \\
& \leq \sum_{i} \sum_{x_{i}<x_{k}^{*} \leq x_{i+1}}\left|\widehat{L}_{t}^{x_{k}^{*}}\right| \\
& =\sum_{-N<x \leq N}\left|\widehat{L}_{t}^{x}\right|<\infty .
\end{aligned}
$$

To see it is of bounded variation in $(t, x)$, consider any partition $D^{\prime} \times D$, where $D^{\prime}=\left\{0=t_{0}<t_{1}<\cdots<t_{n-1}<t_{n}=T\right\}, D=\left\{-N=x_{0}<x_{1}<\right.$ $\left.\cdots<x_{m-1}<x_{m}=N\right\}$

$$
\begin{aligned}
& \sum_{i}\left|h\left(t_{j+1}, x_{i+1}\right)-h\left(t_{j+1}, x_{i}\right)-h\left(t_{j}, x_{i+1}\right)+h\left(t_{j}, x_{i}\right)\right| \\
= & \sum_{i}\left|\sum_{x_{i}<x_{k}^{*} \leq x_{i+1}}\left(\widehat{L}_{t_{j+1}}^{x_{k}^{*}}-\widehat{L}_{t_{j}}^{x_{k}^{*}}\right)\right| \\
\leq & \sum_{i} \sum_{x_{i}<x_{k}^{*} \leq x_{i+1}}\left|\widehat{L}_{t_{j+1}}^{x_{k}^{*}}-\widehat{L}_{t_{j}}^{x_{k}^{*}}\right| \\
= & \sum_{-N<x \leq N}\left|\widehat{L}_{t_{j+1}}^{x}-\widehat{L}_{t_{j}}^{x}\right| .
\end{aligned}
$$

Now applying (2.11) leads to, 


$$
\begin{aligned}
& \sum_{-N<x \leq N}\left|\widehat{L}_{t_{j+1}}^{x}-\widehat{L}_{t_{j}}^{x}\right|=\sum_{-N<x \leq N}\left|\int_{t_{j}}^{t_{j+1}} 1_{\{x\}}\left(X_{s}\right) d V_{s}\right| \\
\leq & \int_{t_{j}}^{t_{j+1}} 1_{[-N, N]}\left(X_{s}\right)\left|d V_{s}\right| .
\end{aligned}
$$

From $(2.15),(2.16)$ and the bounded variation assumption of $V$, we have

$$
\begin{aligned}
& \sum_{j} \sum_{i}\left|h\left(t_{j+1}, x_{i+1}\right)-h\left(t_{j+1}, x_{i}\right)-h\left(t_{j}, x_{i+1}\right)+h\left(t_{j}, x_{i}\right)\right| \\
\leq & \int_{0}^{t} 1_{[-N, N]}\left(X_{s}\right)\left|d V_{s}\right|<\infty .
\end{aligned}
$$

Due to the decomposition (2.13) of local time, the following integral is therefore defined by

$$
\int_{-\infty}^{\infty} f(x) d_{x} L_{t}^{x}=\int_{-\infty}^{\infty} f(x) d_{x} \tilde{L}_{t}^{x}+\int_{-\infty}^{\infty} f(x) d_{x} h(t, x) .
$$

The last integral is a Lebesgue-Stieltjes integral, it doesn't matter whether or not $f$ is continuous as long as it is measurable. If $f$ is of finite $p$-variation $(1 \leq p<2)$, we know the integral $\int_{-\infty}^{\infty} f(x) d_{x} \tilde{L}_{t}^{x}$ is well defined by Young's integration theory.

Remark 2.1 If $f$ belongs to $C^{1}$, we have

$$
\int_{-\infty}^{\infty} f(x) d_{x} L_{t}^{x}=-\int_{-\infty}^{\infty} L_{t}^{x} d f(x) .
$$

This is because $L_{t}$ has a compact support for each $t$, so one can always add some points in the partition to make $L_{t}^{x_{1}}=0$ and $L_{t}^{x_{r}}=0$. So

$$
\begin{aligned}
& \int_{-\infty}^{\infty} f(x) d_{x} L_{t}^{x} \\
= & \lim _{m(D) \rightarrow 0} \sum_{k=1}^{r} f\left(x_{k-1}\right)\left(L_{t}^{x_{k}}-L_{t}^{x_{k-1}}\right) \\
= & \lim _{m(D) \rightarrow 0}\left[\sum_{k=1}^{r} f\left(x_{k-1}\right) L_{t}^{x_{k}}-\sum_{k=0}^{r-1} f\left(x_{k}\right) L_{t}^{x_{k}}\right] \\
= & -\lim _{m(D) \rightarrow 0} \sum_{k=1}^{r}\left(f\left(x_{k}\right)-f\left(x_{k-1}\right)\right) L_{t}^{x_{k}} \\
= & -\int_{-\infty}^{\infty} L_{t}^{x} d f(x) .
\end{aligned}
$$


Assume $g(x)$ is a left continuous function, we use the standard regularizing mollifiers to smoothrize $g$ (e.g. see [14]). Define

$$
\rho(x)= \begin{cases}c \mathrm{e}^{\frac{1}{(x-1)^{2}-1}}, & \text { if } x \in(0,2), \\ 0, & \text { otherwise }\end{cases}
$$

Here $c$ is chosen such that $\int_{0}^{2} \rho(x) d x=1$. Take $\rho_{n}(x)=n \rho(n x)$ as mollifiers. Define

$$
g_{n}(x)=\int_{-\infty}^{+\infty} \rho_{n}(x-y) g(y) d y, \quad n \geq 1 .
$$

Then $g_{n}(x)$ is smooth and

$$
g_{n}(x)=\int_{0}^{2} \rho(z) g\left(x-\frac{z}{n}\right) d z, \quad n \geq 1 .
$$

Using Lebesgue's dominated convergence theorem, one can prove that as $n \rightarrow \infty, g_{n}(x) \rightarrow g(x)$.

Theorem 2.1 Let $g(x)$ be a left continuous function with finite p-variation in $x, 1 \leq p<2, g_{n}(x)$ be defined in (2.20). Then

$$
\int_{-\infty}^{\infty} g_{n}(x) d_{x} \tilde{L}_{t}^{x} \rightarrow \int_{-\infty}^{\infty} g(x) d_{x} \tilde{L}_{t}^{x} \text {, as } n \rightarrow \infty .
$$

Proof: Let $\delta>0$ satisfy $\frac{1}{2+\delta}+\frac{1}{p}>1$. From Lemma 2.1, $\tilde{L}_{t}^{x}$ is of bounded $(2+\delta)$-variation in $x$ uniformly in $t$. From [31], $g(x)$ being of bounded $p$ variation, $1 \leq p<2$, is equivalent to that for any partition $D:=D_{-N, N}=$ $\left\{-N=x_{0}<x_{1}<\cdots<x_{r}=N\right\}$ defined as before, there is an increasing function $w$ such that

$$
\left|g\left(x_{l+1}\right)-g\left(x_{l}\right)\right| \leq\left(w\left(x_{l+1}\right)-w\left(x_{l}\right)\right)^{\frac{1}{p}}, \forall x_{l}, x_{l+1} \in D,
$$

where $w(x)$ is the total $p$-variation of $f$ in the interval $[-N-2, x]$. Using Hölder inequality, we get

$$
\begin{aligned}
& \sup _{D} \sum_{l=1}^{r}\left|g_{n}\left(x_{l}\right)-g_{n}\left(x_{l-1}\right)\right|^{p} \\
= & \sup _{D} \sum_{l=1}^{r}\left|\int_{0}^{2} \rho(z)\left[g\left(x_{l}-\frac{z}{n}\right)-g\left(x_{l-1}-\frac{z}{n}\right)\right] d z\right|^{p} \\
\leq & M_{1} \sup _{D} \sum_{l=1}^{r}\left(\int_{0}^{2}\left|g\left(x_{l}-\frac{z}{n}\right)-g\left(x_{l-1}-\frac{z}{n}\right)\right|^{p} d z\right) \\
\leq & M_{1} \int_{0}^{2} \sup _{D} \sum_{l=1}^{r}\left|g\left(x_{l}-\frac{z}{n}\right)-g\left(x_{l-1}-\frac{z}{n}\right)\right|^{p} d z \\
\leq & M_{1} \int_{0}^{2}\left(w\left(N-\frac{z}{n}\right)-w\left(-N-\frac{z}{n}\right)\right) d z,
\end{aligned}
$$


where $M_{1}$ is a constant. As

$$
w\left(N-\frac{z}{n}\right)-w\left(-N-\frac{z}{n}\right) \leq w(N)
$$

so

$$
\sup _{D} \sum_{l=1}^{r}\left|g_{n}\left(x_{l}\right)-g_{n}\left(x_{l-1}\right)\right|^{p} \leq 2 M_{1} w(N)<\infty
$$

which means that $g_{n}(x)$ is of bounded $p$-variation in $x$ uniformly in $n$. Then (2.21) follows from Young's ([30] or [31]) convergence theorem we can get the result directly.

Remark 2.2 From the Lebesgue's dominated convergence theorem, for $g$ in the above theorem, we know

$$
\int_{-\infty}^{\infty} g_{n}(x) d_{x} h(t, x) \rightarrow \int_{-\infty}^{\infty} g(x) d_{x} h(t, x), \text { as } n \rightarrow \infty .
$$

With Theorem 2.1, it follows that

$$
\int_{-\infty}^{\infty} g_{n}(x) d_{x} L_{t}^{x} \rightarrow \int_{-\infty}^{\infty} g(x) d_{x} L_{t}^{x} \text {, as } n \rightarrow \infty .
$$

Using the above theorem, we can get an extension of Itô's Formula.

Theorem 2.2 Let $X=\left(X_{t}\right)_{t \geq 0}$ be a continuous semi-martingale and $f$ : $R \rightarrow R$ be a left continuous, locally bounded function and have left derivative $\nabla^{-} f(x)$ being left continuous and locally bounded. Assume $\nabla^{-} f(x)$ is of bounded q-variation, where $1 \leq q<2$. Then we have the following changeof-variable formula

$$
f\left(X_{t}\right)=f\left(X_{0}\right)+\int_{0}^{t} \nabla^{-} f\left(X_{s}\right) d X_{s}-\int_{-\infty}^{\infty} \nabla^{-} f(x) d_{x} L_{t}^{x},
$$

where $L_{t}^{x}$ is the local time of $X_{t}$ at $x$.

Proof: The integral $\int_{-\infty}^{\infty} \nabla^{-} f(x) d_{x} L_{t}^{x}$ is defined pathwise as a combination of rough path integral and Lebesgue-Stieltjes integral. We may quote the proof in [14] and define

$$
f_{n}(x)=\int_{-\infty}^{+\infty} \rho_{n}(x-y) f(y) d y, \quad n \geq 1
$$

The convergence of all terms except the second order derivative term are the same as in the proof in [14]. By occupation times formula and Remark 2.1, the second order derivative term is 


$$
\begin{aligned}
\frac{1}{2} \int_{0}^{t} \Delta f_{n}\left(X_{s}\right) d<M>_{s} & =\int_{-\infty}^{\infty} \Delta f_{n}(x) L_{t}^{x} d x \\
& =\int_{-\infty}^{\infty} L_{t}^{x} d \nabla f_{n}(x) \\
& =-\int_{-\infty}^{\infty} \nabla f_{n}(x) d_{x} L_{t}^{x} .
\end{aligned}
$$

It follows from (2.23) that,

$$
\frac{1}{2} \int_{0}^{t} \Delta f_{n}\left(X_{s}\right) d<M>_{s} \rightarrow-\int_{-\infty}^{\infty} \nabla^{-} f(x) d_{x} L_{t}^{x}
$$

when $n \rightarrow \infty$. Our claim is asserted.

Needless to say, there are many cases that Theorem 2.2 works, but other extensions of Itô's formula do not apply immediately. The following is an obvious example:

Example 2.1 Consider a function $f(x)=x^{3} \cos \frac{1}{x}$ for $x \neq 0$ and $f(0)=0$. This function is $C^{1}$ and its derivative is $f^{\prime}(x)=3 x^{2} \cos \frac{1}{x}+x \sin \frac{1}{x}$ for $x \neq 0$ and $f^{\prime}(0)=0$. It is easy to see that $f^{\prime}$ is not of bounded variation, but of $p$-variation for any $p>1$ (see Example 3.1 for a proof in a more complicated case). So Theorem 2.2 can be used, while Meyer's formula cannot apply to this situation.

\section{Two-parameter $p, q$-variation path integrals}

In this section, the following notations are used: $\Phi(u), \Psi(u), \Phi_{1}(u), \Psi_{1}(u)$ denote continuous functions strictly increasing from 0 to $\infty$ with $\mathrm{u}$, where $u \geq 0$ is a variable, and $\Phi(0), \Psi(0), \Phi_{1}(0), \Psi_{1}(0) \equiv 0 ; \varphi(u), \psi(u), \varphi_{1}(u)$, $\psi_{1}(u)$ denote the inverse functions of $\Phi(u), \Psi(u), \Phi_{1}(u), \Psi_{1}(u)$, respectively; $\omega, \chi$ are monotone increasing functions of one variable, $x$ or $y$.

Firstly in the following we will define a two-parameter Young integral $\int_{x^{\prime}}^{x^{\prime \prime}} \int_{y^{\prime}}^{y^{\prime \prime}} F(x, y) d_{x, y} G(x, y)$. We will use some idea from Young [31]. But Young's condition is very strong and the class of functions that satisfy Young's condition is restricted. In particular, Young's condition does not seem to include the class of functions of bounded variation and many important examples. We give a new and weaker condition for the integration in this section. We will use Lyons' idea of control functions to simplify our proof. One can see our condition is a natural extension of locally bounded multi-dimensional L-S measure. First, if $F(x, y)$ is a simple function, say

$$
F(x, y)=\sum_{i=1}^{M} \sum_{j=1}^{M^{\prime}} F\left(x_{i-1}, y_{j-1}\right) 1_{\left\{x_{i-1}<x \leq x_{i}, y_{j-1}<y \leq y_{j}\right\}},
$$


as normal we can see that the integral of the simple function can be defined as

$$
\begin{aligned}
& \int_{x^{\prime}}^{x^{\prime \prime}} \int_{y^{\prime}}^{y^{\prime \prime}} F(x, y) d_{x, y} G(x, y) \\
= & \sum_{i=1}^{M} \sum_{j=1}^{M^{\prime}} F\left(x_{i-1}, y_{j-1}\right)\left(G\left(x_{i}, y_{j}\right)-G\left(x_{i-1}, y_{j}\right)-G\left(x_{i}, y_{j-1}\right)+G\left(x_{i-1}, y_{j-1}\right)\right) .
\end{aligned}
$$

Before we proceed, we need the following definition.

Definition 3.1 Let $E \times E^{\prime}:=\left\{x^{\prime}=x_{0}<x_{1}<\cdots<x_{N}=x^{\prime \prime}, y^{\prime}=y_{0}<\right.$ $\left.y_{1}<\cdots<y_{N^{\prime}}=y^{\prime \prime}\right\}$ be an arbitrary partition of $\left[x^{\prime}, x^{\prime \prime}\right] \times\left[y^{\prime}, y^{\prime \prime}\right]$. We call $F(x, y)$ is of bounded $\Phi$-variation in $x$ uniformly in $y$, if

$$
\sup _{y \in\left[y^{\prime}, y^{\prime \prime}\right]} \sup _{E} \sum_{k=1}^{N} \Phi\left(\left|F\left(x_{k}, y\right)-F\left(x_{k-1}, y\right)\right|\right)<\infty .
$$

We call $G(x, y)$ is of bounded $\Phi_{1}, \Psi_{1}$-variation in $(x, y)$, if

$$
\sup _{E \times E^{\prime}} \sum_{j=1}^{N^{\prime}} \Psi_{1}\left(\sum_{i=1}^{N} \Phi_{1}\left(\left|\Delta_{i} \Delta_{j} G\right|\right)\right)<\infty
$$

where

$$
\Delta_{i} \Delta_{j} G:=G\left(x_{i}, y_{j}\right)-G\left(x_{i-1}, y_{j}\right)-G\left(x_{i}, y_{j-1}\right)+G\left(x_{i-1}, y_{j-1}\right) .
$$

If $\Psi_{1}(u)=u$, we call $G(x, y)$ is of bounded $\Phi_{1}$-variation in $(x, y)$. If $\Phi_{1}(u)=$ $u^{p}, \Psi_{1}(u)=u^{q}, p, q \geq 1$, we call $G(x, y)$ is of bounded $p, q$-variation in $(x, y)$.

In the following, we will give an example of $p, 1$-variation $(p>1)$ function.

Example 3.1 Consider

$$
f(x, y)=x y \sin \left(\frac{1}{x}+\frac{1}{y}\right), 0<x, y \leq 1, f(0, y)=f(x, 0)=f(0,0)=0 .
$$

This is a continuous function of unbounded variation but of bounded $p, 1$ variation $(p>1)$. To see it is of unbounded variation, we take the partition $E_{1} \times E_{2}=\left\{0<\frac{1}{n \pi+\frac{\pi}{2}-1}<\frac{1}{n \pi-1}<\cdots<\frac{1}{\pi-1}<1,0<1\right\}$,

$$
\begin{aligned}
& \sum_{i, j}\left|\Delta_{i} \Delta_{j} f\right|=\sum_{i}\left|x_{i} \sin \left(\frac{1}{x_{i}}+1\right)-x_{i-1} \sin \left(\frac{1}{x_{i-1}}+1\right)\right| \\
= & \sum_{i}\left|\frac{1}{i \pi+\frac{\pi}{2}-1} \sin \left(i \pi+\frac{\pi}{2}\right)-\frac{1}{i \pi-1} \sin (i \pi)\right| \\
= & \sum_{i} \frac{1}{i \pi+\frac{\pi}{2}-1} \\
= & \infty .
\end{aligned}
$$


To see it is of bounded $p, 1$-variation for any $p>1$, consider any partition $E \times E^{\prime}$

$$
\begin{aligned}
& \sum_{i, j}\left|\Delta_{i} \Delta_{j} f\right|^{p} \\
& =\sum_{i, j} \mid x_{i} y_{j} \sin \left(\frac{1}{x_{i}}+\frac{1}{y_{j}}\right)-x_{i-1} y_{j} \sin \left(\frac{1}{x_{i-1}}+\frac{1}{y_{j}}\right) \\
& -x_{i} y_{j-1} \sin \left(\frac{1}{x_{i}}+\frac{1}{y_{j-1}}\right)+\left.x_{i-1} y_{j-1} \sin \left(\frac{1}{x_{i-1}}+\frac{1}{y_{j-1}}\right)\right|^{p} \\
& =\sum_{i, j} \mid y_{j}\left[x_{i} \sin \left(\frac{1}{x_{i}}+\frac{1}{y_{j}}\right)-x_{i-1} \sin \left(\frac{1}{x_{i-1}}+\frac{1}{y_{j}}\right)\right] \\
& -\left.y_{j-1}\left[x_{i} \sin \left(\frac{1}{x_{i}}+\frac{1}{y_{j-1}}\right)-x_{i-1} \sin \left(\frac{1}{x_{i-1}}+\frac{1}{y_{j-1}}\right)\right]\right|^{p} \\
& =\sum_{i, j} \mid y_{j}\left(x_{i}-x_{i-1}\right) \sin \left(\frac{1}{x_{i}}+\frac{1}{y_{j}}\right)+y_{j} x_{i-1}\left[\sin \left(\frac{1}{x_{i}}+\frac{1}{y_{j}}\right)-\sin \left(\frac{1}{x_{i-1}}+\frac{1}{y_{j}}\right)\right] \\
& -y_{j-1}\left(x_{i}-x_{i-1}\right) \sin \left(\frac{1}{x_{i}}+\frac{1}{y_{j-1}}\right) \\
& -\left.y_{j-1} x_{i-1}\left[\sin \left(\frac{1}{x_{i}}+\frac{1}{y_{j-1}}\right)-\sin \left(\frac{1}{x_{i-1}}+\frac{1}{y_{j-1}}\right)\right]\right|^{p} \\
& =\sum_{i, j} \mid\left(x_{i}-x_{i-1}\right)\left[y_{j} \sin \left(\frac{1}{x_{i}}+\frac{1}{y_{j}}\right)-y_{j-1} \sin \left(\frac{1}{x_{i}}+\frac{1}{y_{j-1}}\right)\right] \\
& +x_{i-1}\left[y _ { j } \left(\left(\sin \left(\frac{1}{x_{i}}+\frac{1}{y_{j}}\right)-\sin \left(\frac{1}{x_{i-1}}+\frac{1}{y_{j}}\right)\right)\right.\right. \\
& \left.-y_{j-1}\left(\sin \left(\frac{1}{x_{i}}+\frac{1}{y_{j-1}}\right)-\sin \left(\frac{1}{x_{i-1}}+\frac{1}{y_{j-1}}\right)\right)\right]\left.\right|^{p} \\
& =\sum_{i, j} \mid\left(x_{i}-x_{i-1}\right)\left[\left(y_{j}-y_{j-1}\right) \sin \left(\frac{1}{x_{i}}+\frac{1}{y_{j}}\right)\right. \\
& \left.+y_{j-1}\left(\sin \left(\frac{1}{x_{i}}+\frac{1}{y_{j}}\right)-\sin \left(\frac{1}{x_{i}}+\frac{1}{y_{j-1}}\right)\right)\right] \\
& +x_{i-1}\left[\left(y_{j}-y_{j-1}\right)\left(\sin \left(\frac{1}{x_{i}}+\frac{1}{y_{j}}\right)-\sin \left(\frac{1}{x_{i-1}}+\frac{1}{y_{j}}\right)\right)\right. \\
& +y_{j-1}\left(\sin \left(\frac{1}{x_{i}}+\frac{1}{y_{j}}\right)-\sin \left(\frac{1}{x_{i-1}}+\frac{1}{y_{j}}\right)\right. \\
& \left.\left.-\sin \left(\frac{1}{x_{i}}+\frac{1}{y_{j-1}}\right)+\sin \left(\frac{1}{x_{i-1}}+\frac{1}{y_{j-1}}\right)\right)\right]\left.\right|^{p} \\
& \leq c_{p}\left\{\sum_{i, j}\left|\left(x_{i}-x_{i-1}\right)\left(y_{j}-y_{j-1}\right) \sin \left(\frac{1}{x_{i}}+\frac{1}{y_{j}}\right)\right|^{p}\right.
\end{aligned}
$$




$$
\left.\begin{array}{l}
+\sum_{i, j}\left|y_{j-1}\left(x_{i}-x_{i-1}\right)\left(\sin \left(\frac{1}{x_{i}}+\frac{1}{y_{j}}\right)-\sin \left(\frac{1}{x_{i}}+\frac{1}{y_{j-1}}\right)\right)\right|^{p} \\
+\sum_{i, j}\left|x_{i-1}\left(y_{j}-y_{j-1}\right)\left(\sin \left(\frac{1}{x_{i}}+\frac{1}{y_{j}}\right)-\sin \left(\frac{1}{x_{i-1}}+\frac{1}{y_{j}}\right)\right)\right|^{p} \\
+\sum_{i, j} \mid x_{i-1} y_{j-1}\left(\sin \left(\frac{1}{x_{i}}+\frac{1}{y_{j}}\right)-\sin \left(\frac{1}{x_{i-1}}+\frac{1}{y_{j}}\right)\right. \\
\left.\quad-\sin \left(\frac{1}{x_{i}}+\frac{1}{y_{j-1}}\right)+\sin \left(\frac{1}{x_{i-1}}+\frac{1}{y_{j-1}}\right)\right)\left.\right|^{p}
\end{array}\right\}
$$

where $c_{p}$ is a constant. It's easy to see that

$$
I \leq \sum_{i, j}\left(x_{i}-x_{i-1}\right)^{p}\left(y_{j}-y_{j-1}\right)^{p} \leq 1 .
$$

For $I I$, as $|\sin x| \leq x$, so

$$
\begin{aligned}
I I & \leq 2^{p-1} \sum_{i, j} y_{j-1}^{p}\left(x_{i}-x_{i-1}\right)^{p}\left|\sin \left(\frac{1}{x_{i}}+\frac{1}{y_{j}}\right)-\sin \left(\frac{1}{x_{i}}+\frac{1}{y_{j-1}}\right)\right| \\
& =2^{p-1} \sum_{i, j} y_{j-1}^{p}\left(x_{i}-x_{i-1}\right)^{p} \cdot\left|2 \cos \frac{\frac{2}{x_{i}}+\frac{1}{y_{j}}+\frac{1}{y_{j-1}}}{2} \sin \frac{\frac{1}{y_{j}}-\frac{1}{y_{j-1}}}{2}\right| \\
& \leq 2^{p-1} \sum_{i, j} y_{j-1}^{p}\left(x_{i}-x_{i-1}\right)^{p}\left(\frac{1}{y_{j-1}}-\frac{1}{y_{j}}\right) \\
& =2^{p-1} \sum_{i}\left(x_{i}-x_{i-1}\right)^{p} \sum_{j} y_{j-1}^{p}\left(\frac{1}{y_{j-1}}-\frac{1}{y_{j}}\right) .
\end{aligned}
$$

It is obvious that

$$
\sum_{i}\left(x_{i}-x_{i-1}\right)^{p}<\infty .
$$

And also because

$$
\begin{aligned}
\sum_{j} y_{j-1}^{p}\left(\frac{1}{y_{j-1}}-\frac{1}{y_{j}}\right) & \leq \sum_{j} y_{j-1}^{p} \frac{y_{j}-y_{j-1}}{y_{j-1}^{2}} \\
& =\sum_{j} y_{j-1}^{p-2}\left(y_{j}-y_{j-1}\right) \\
& \leq \int_{0}^{1} y^{p-2} d y=\frac{1}{p-1}
\end{aligned}
$$

So we get $I I<\infty$. Similar to the discussion of $I I$, we can also prove that $I I I<\infty$. About IV, 


$$
\begin{aligned}
& \text { IV } \\
& =\sum_{i, j} x_{i-1}^{p} y_{j-1}^{p} \mid 2 \cos \frac{\frac{1}{x_{i}}+\frac{1}{x_{i-1}}+\frac{2}{y_{j}}}{2} \sin \frac{\frac{1}{x_{i}}-\frac{1}{x_{i-1}}}{2} \\
& -\left.2 \cos \frac{\frac{1}{x_{i}}+\frac{1}{x_{i-1}}+\frac{2}{y_{j-1}}}{2} \sin \frac{\frac{1}{x_{i}}-\frac{1}{x_{i-1}}}{2}\right|^{p} \\
& =2^{p} \sum_{i, j} x_{i-1}^{p} y_{j-1}^{p}\left|\sin \frac{\frac{1}{x_{i}}-\frac{1}{x_{i-1}}}{2}\right|^{p} \cdot\left|\cos \frac{\frac{1}{x_{i}}+\frac{1}{x_{i-1}}+\frac{2}{y_{j}}}{2}-\cos \frac{\frac{1}{x_{i}}+\frac{1}{x_{i-1}}+\frac{2}{y_{j-1}}}{2}\right|^{p} \\
& =2^{p} \sum_{i, j} x_{i-1}^{p} y_{j-1}^{p}\left|\sin \frac{\frac{1}{x_{i}}-\frac{1}{x_{i-1}}}{2}\right|^{p} \\
& \cdot\left|-2 \sin \frac{\frac{1}{x_{i}}+\frac{1}{x_{i-1}}+\frac{1}{y_{j}}+\frac{1}{y_{j-1}}}{2} \sin \frac{\frac{1}{y_{j}}-\frac{1}{y_{j-1}}}{2}\right|^{p} \\
& \leq 2^{p} \cdot 2^{p} \sum_{i, j} x_{i-1}^{p} y_{j-1}^{p} \frac{1}{2}\left(\frac{1}{x_{i-1}}-\frac{1}{x_{i}}\right) \cdot \frac{1}{2}\left(\frac{1}{y_{j-1}}-\frac{1}{y_{j}}\right) \\
& =2^{2 p-2} \sum_{i} x_{i-1}^{p}\left(\frac{1}{x_{i-1}}-\frac{1}{x_{i}}\right) \sum_{j} y_{j-1}^{p}\left(\frac{1}{y_{j-1}}-\frac{1}{y_{j}}\right) \\
& \leq \frac{2^{2 p-2}}{(p-1)^{2}},
\end{aligned}
$$

following from a similar argument as in (3.4). So the function $f(x, y)=$ xysin $\left(\frac{1}{x}+\frac{1}{y}\right), 0<x, y \leq 1, f(0, y)=f(x, 0)=f(0,0)=0$, is of bounded $p, 1$-variation for any $p>1$. Moreover, from the above proof, we can see for this function $f(x, y)$ on $(x, y) \in\left[0, \delta_{1}\right] \times\left[0, \delta_{2}\right]$, its $p, 1$-variation tends to 0 when either $\delta_{1}$ or $\delta_{2}$ decreases to 0 .

We say a function $f(x, y)$ has a jump at $\left(x_{1}, y_{1}\right)$ if there exists an $\varepsilon>0$ such that for any $\delta>0$, there exists $\left(x_{2}, y_{2}\right)$ satisfying $\max \left\{\left|x_{1}-x_{2}\right|, \mid y_{1}-\right.$ $\left.y_{2} \mid\right\}<\delta$ and $\left|f\left(x_{2}, y_{2}\right)-f\left(x_{1}, y_{2}\right)-f\left(x_{2}, y_{1}\right)+f\left(x_{1}, y_{1}\right)\right|>\varepsilon$. For a function $G(x, y)$ of bounded $\Phi_{1}, \Psi_{1}$-variation, for any given $\varepsilon>0$, it is easy to see that there exists a $\delta(\varepsilon)>0$ and a finite number of jump points $\left\{\left(x_{1}, y_{1}\right), \cdots,\left(x_{n_{0}}, y_{m_{0}}\right)\right\}$ such that $|G(x, y)-G(x, \tilde{y})-G(\tilde{x}, y)+G(\tilde{x}, \tilde{y})|<\varepsilon$ whenever $\max \{|\tilde{x}-x|,|\tilde{y}-y|\}<\delta(\varepsilon),[\tilde{x}, x] \cap\left\{x_{1}, \cdots, x_{n_{0}}\right\}=\emptyset$ and $[\tilde{y}, y] \cap\left\{y_{1}, \cdots, y_{m_{0}}\right\}=\emptyset$. Denote $H_{0} \times H_{0}^{\prime}:=\left\{x_{1}, \cdots, x_{n_{0}}\right\} \times\left\{y_{1}, \cdots, y_{m_{0}}\right\}$. In the following, we assume the following finite large jump condition: for any $\varepsilon>0$, there exists at most finite many points $\left\{x_{1}, \cdots, x_{n_{1}}\right\},\left\{y_{1}, \cdots, y_{m_{1}}\right\}$ and a constant $\delta(\varepsilon)>0$ such that the total $\Phi_{1}, \Psi_{1}$-variation of $G$ on $[x, x+\delta] \times\left[y^{\prime}, y^{\prime \prime}\right]$ is smaller than $\varepsilon$ if $[x, x+\delta] \cap\left\{x_{1}, \cdots, x_{n_{1}}\right\}=\emptyset$, and the total $\Phi_{1}, \Psi_{1}$-variation of $G$ on $\left[x^{\prime}, x^{\prime \prime}\right] \times[y, y+\delta]$ is smaller than $\varepsilon$ if $[y, y+\delta] \cap\left\{y_{1}, \cdots, y_{m_{1}}\right\}=\emptyset$. Denote $H \times H^{\prime}:=\left\{x_{1}, \cdots, x_{n_{1}}\right\} \times\left\{y_{1}, \cdots, y_{m_{1}}\right\}$. It is obviously that $H \times H^{\prime} \supset H_{0} \times H_{0}^{\prime}$. There are many examples of bounded $\Phi_{1}, \Psi_{1}$-variation functions that satisfy the finite large jump condition. But it 
is not clear whether or not the bounded $\Phi_{1}, \Psi_{1}$-variation condition implies automatically the finite large jump condition in the two parameters case although this is true in the one parameter case.

Denote by $\omega\left(x_{k}\right)$ the total uniform $\Phi$-variation of $F$ in $x$ in the interval $\left[x^{\prime}, x_{k}\right]$ and $\chi\left(y_{l}\right)$ the total uniform $\Psi$-variation of $F$ in $y$ in the interval $\left[y^{\prime}, y_{l}\right]$. For the partition $E \times E^{\prime}$, denote by $m\left(E \times E^{\prime}\right)$ the mesh of the partition.

We need the following simple inequalities: Let $f$ be a nonnegative and nondecreasing function, then

$$
\sum_{p=0}^{\infty} 2^{p-1} f\left(\frac{1}{2^{p}}\right) \leq \sum_{m=1}^{\infty} f\left(\frac{1}{m}\right) \leq \sum_{p=0}^{\infty} 2^{p} f\left(\frac{1}{2^{p}}\right)
$$

and for any $v \geq 1$,

$$
\sum_{p=v}^{\infty} 2^{p-1} f\left(\frac{1}{2^{p}}\right) \leq \sum_{m=2^{v-1}+1}^{\infty} f\left(\frac{1}{m}\right) \leq \sum_{p=v-1}^{\infty} 2^{p} f\left(\frac{1}{2^{p}}\right),
$$

if the series $\sum_{m=1}^{\infty} f\left(\frac{1}{m}\right)$ is convergent. These inequalities were also used in the proof of Young main results. We listed them here only for the purpose to make the proof of the following theorem easier to understand. The proof is elementary and omitted.

Theorem 3.1 Let $F(x, y)$ be a continuous function of bounded $\Phi$-variation in $x$ uniformly in $y$, and be of bounded $\Psi$-variation in $y$ uniformly in $x$; $G(x, y)$ be of bounded $\Phi_{1}, \Psi_{1}$-variation in $(x, y)$ and satisfy the finite large jump condition, where $\Psi_{1}, \Phi_{1}$ are convex functions. If there exist monotone increasing functions $\varrho$ and $\sigma$ subject to $\varrho(u) \sigma(u)=u$ such that

$$
\sum_{m, n} \varrho\left[\varphi\left(\frac{1}{n}\right)\right] \sigma\left[\psi\left(\frac{1}{m}\right)\right] \varphi_{1}\left[\frac{1}{n} \psi_{1}\left(\frac{1}{m}\right)\right]<\infty,
$$

then the integral

$$
\begin{aligned}
& \int_{x^{\prime}}^{x^{\prime \prime}} \int_{y^{\prime}}^{y^{\prime \prime}} F(x, y) d_{x, y} G(x, y) \\
= & \lim _{m\left(E \times E^{\prime}\right) \rightarrow 0} \sum_{i=1}^{N} \sum_{j=1}^{N^{\prime}} F\left(x_{i-1}, y_{j-1}\right) \Delta_{i} \Delta_{j} G
\end{aligned}
$$

is well defined with all partitions $E \times E^{\prime}$ of $\left[x^{\prime}, x^{\prime \prime}\right] \times\left[y^{\prime}, y^{\prime \prime}\right]$ including suitable finite sets $H \times H^{\prime}$ as defined above, i.e. for any given $\varepsilon>0$, we can determine finite sets $H$ and $H^{\prime}$ of variables $x$ and $y$ respectively such that

$$
\left|\int_{x^{\prime}}^{x^{\prime \prime}} \int_{y^{\prime}}^{y^{\prime \prime}} F(x, y) d_{x, y} G(x, y)-\sum_{i=1}^{N} \sum_{j=1}^{N^{\prime}} F\left(x_{i-1}, y_{j-1}\right) \Delta_{i} \Delta_{j} G\right|<\varepsilon
$$


as soon as the partition $E=\left\{x^{\prime}=x_{1}<x_{2}<\cdots<x_{N}=x^{\prime \prime}\right\}$ and $E^{\prime}=$ $\left\{y^{\prime}=y_{1}<y_{2}<\cdots<y_{N^{\prime}}=y^{\prime \prime}\right\}$ includes $H$ and $H^{\prime}$ among their points of divisions respectively.

Proof: For any partition $E \times E^{\prime}:=\left\{x^{\prime}=x_{0}<x_{1}<\cdots<x_{N}=x^{\prime \prime}, y^{\prime}=\right.$ $\left.y_{0}<y_{1}<\cdots<y_{N^{\prime}}=y^{\prime \prime}\right\}$, consider

$$
F_{E, E^{\prime}}(x, y):=\sum_{i=1}^{N} \sum_{j=1}^{N^{\prime}} F\left(x_{i-1}, y_{j-1}\right) 1_{\left\{x_{i-1} \leq x<x_{i}, y_{j-1} \leq y<y_{j}\right\}},
$$

then

$$
\begin{aligned}
S\left(E, E^{\prime}\right) & :=S_{F_{E, E^{\prime}}}\left(E, E^{\prime}\right):=\int_{x^{\prime}}^{x^{\prime \prime}} \int_{y^{\prime}}^{y^{\prime \prime}} F_{E, E^{\prime}}(x, y) d_{x, y} G(x, y) \\
& =\sum_{i=1}^{N} \sum_{j=1}^{N^{\prime}} F\left(x_{i-1}, y_{j-1}\right) \Delta_{i} \Delta_{j} G
\end{aligned}
$$

From the assumption of $F$, let $P, Q$ be the total $\Phi$ - and $\Psi$-variation of $F$ in $x$ and $y$ respectively, so

$$
\begin{aligned}
& \sum_{k=1}^{N} \Phi\left(\left|F\left(x_{k}, y\right)-F\left(x_{k-1}, y\right)\right|\right) \leq P, \\
& \sum_{l=1}^{N^{\prime}} \Psi\left(\left|F\left(x, y_{l}\right)-F\left(x, y_{l-1}\right)\right|\right) \leq Q,
\end{aligned}
$$

which are equivalent to

$$
\begin{array}{r}
\left|F\left(x_{k}, y\right)-F\left(x_{k-1}, y\right)\right| \leq \varphi\left(\omega\left(x_{k}\right)-\omega\left(x_{k-1}\right)\right), \quad k=1,2, \cdots, N \\
\left|F\left(x, y_{l}\right)-F\left(x, y_{l-1}\right)\right| \leq \psi\left(\chi\left(y_{l}\right)-\chi\left(y_{l-1}\right)\right), \quad l=1,2, \cdots, N^{\prime} .
\end{array}
$$

Obviously, if $y_{j-1} \leq y<y_{j}, j=1, \cdots, N^{\prime}$,

$$
\begin{aligned}
& \left|F_{E, E^{\prime}}\left(x_{k}, y\right)-F_{E, E^{\prime}}\left(x_{k-1}, y\right)\right| \\
= & \left|F\left(x_{k}, y_{j-1}\right)-F\left(x_{k-1}, y_{j-1}\right)\right| \\
\leq & \varphi\left(\omega\left(x_{k}\right)-\omega\left(x_{k-1}\right)\right), \quad k=1,2, \cdots, N,
\end{aligned}
$$

and if $x_{i-1} \leq x<x_{i}, i=1, \cdots, N$,

$$
\begin{aligned}
& \left|F_{E, E^{\prime}}\left(x, y_{l}\right)-F_{E, E^{\prime}}\left(x, y_{l-1}\right)\right| \\
= & \left|F\left(x_{i-1}, y_{l}\right)-F\left(x_{i-1}, y_{l-1}\right)\right| \\
\leq & \psi\left(\chi\left(y_{l}\right)-\chi\left(y_{l-1}\right)\right), \quad l=1,2, \cdots, N^{\prime} .
\end{aligned}
$$

Because $\omega$ and $\chi$ are both increasing functions, so we can define a sequence of finite sets: 


$$
\begin{aligned}
& E_{p}: \omega\left(x^{(2)}\right)-\omega\left(x^{(0)}\right) \leq 2^{-p} P, \\
& E_{q}^{\prime}: \chi\left(y^{(2)}\right)-\chi\left(y^{(0)}\right) \leq 2^{-q} Q,
\end{aligned}
$$

where $x^{(0)}, x^{(2)}$ are two consecutive points of $E_{p}, y^{(0)}, y^{(2)}$ are consecutive points of $E_{q}^{\prime}$. Such $E_{p}$ and $E_{q}^{\prime}$ can be defined by induction as for each $p \geq 0$ on $E_{p}$ either $\omega\left(x^{(2)}\right)-\omega\left(x^{(0)}\right) \leq 2^{-(p+1)} P$ or $2^{-(p+1)} P<\omega\left(x^{(2)}\right)-\omega\left(x^{(0)}\right) \leq$ $2^{-p} P$. In the latter case, we insert one point between such a pair to get $E_{p+1}$ (we insert at most $2^{p}$ points) such that

$$
E_{p+1}: \omega\left(x^{(2)}\right)-\omega\left(x^{(1)}\right) \leq 2^{-(p+1)} P,
$$

where $x^{(1)}, x^{(2)}$ are consecutive points of $E_{p+1}$. In the same way, we can get $E_{q+1}^{\prime}$ such that

$$
E_{q+1}^{\prime}: \chi\left(y^{(2)}\right)-\chi\left(y^{(1)}\right) \leq 2^{-(q+1)} Q
$$

where $y^{(1)}, y^{(2)}$ are consecutive points of $E_{q+1}^{\prime}$. In $E_{p+1}$, there are at most $2^{p+1}$ points and in $E_{q+1}^{\prime}$, there are at most $2^{q+1}$ points. We will prove our theorem in four steps.

Step 1: Note

$$
\begin{aligned}
& S\left(E_{p+1}, E_{q+1}^{\prime}\right)-S\left(E_{p}, E_{q+1}^{\prime}\right)-S\left(E_{p+1}, E_{q}^{\prime}\right)+S\left(E_{p}, E_{q}^{\prime}\right) \\
&= \sum_{i=1,3,5 \cdots, 2^{p+1}-1} \sum_{j=1,3,5 \cdots, 2^{q+1}-1}\left[F\left(x_{i-1}, y_{j-1}\right) \Delta_{i} \Delta_{j} G\right. \\
&+ F\left(x_{i-1}, y_{j}\right) \Delta_{i} \Delta_{j+1} G+F\left(x_{i}, y_{j}\right) \Delta_{i+1} \Delta_{j+1} G+F\left(x_{i}, y_{j-1}\right) \Delta_{i+1} \Delta_{j} G \\
&-F\left(x_{i-1}, y_{j-1}\right)\left(G\left(x_{i+1}, y_{j}\right)-G\left(x_{i-1}, y_{j}\right)\right. \\
&\left.-G\left(x_{i+1}, y_{j-1}\right)+G\left(x_{i-1}, y_{j-1}\right)\right) \\
&-F\left(x_{i-1}, y_{j}\right)\left(G\left(x_{i+1}, y_{j+1}\right)-G\left(x_{i+1}, y_{j}\right)\right.\left.-G\left(x_{i-1}, y_{j+1}\right)+G\left(x_{i-1}, y_{j}\right)\right) \\
&-F\left(x_{i-1}, y_{j-1}\right)\left(G\left(x_{i}, y_{j+1}\right)-G\left(x_{i-1}, y_{j+1}\right)\right.\left.-G\left(x_{i}, y_{j-1}\right)+G\left(x_{i-1}, y_{j-1}\right)\right) \\
&-F\left(x_{i}, y_{j-1}\right)\left(G\left(x_{i+1}, y_{j+1}\right)-G\left(x_{i+1}, y_{j-1}\right)-G\left(x_{i}, y_{j+1}\right)+G\left(x_{i}, y_{j-1}\right)\right) \\
&+F\left(x_{i-1}, y_{j-1}\right)\left(G\left(x_{i+1}, y_{j+1}\right)-G\left(x_{i+1}, y_{j-1}\right)\right.
\end{aligned}
$$




$$
=\sum_{i=1,3,5 \cdots, 2^{p+1}-1} \sum_{j=1,3,5 \cdots, 2^{q+1}-1}\left(\Delta_{i} \Delta_{j} F\right)\left(\Delta_{i+1} \Delta_{j+1} G\right) .
$$

Because

$$
\begin{aligned}
\left|\Delta_{i} \Delta_{j} F\right| & \leq\left|F\left(x_{i}, y_{j}\right)-F\left(x_{i-1}, y_{j}\right)\right|+\left|F\left(x_{i}, y_{j-1}\right)-F\left(x_{i-1}, y_{j-1}\right)\right| \\
& \leq 2 \varphi\left(2^{-(p+1)} P\right) \leq 2 \varphi\left(2^{-p} P\right)
\end{aligned}
$$

and also

$$
\begin{aligned}
\left|\Delta_{i} \Delta_{j} F\right| & \leq\left|F\left(x_{i}, y_{j}\right)-F\left(x_{i}, y_{j-1}\right)\right|+\left|F\left(x_{i-1}, y_{j}\right)-F\left(x_{i-1}, y_{j-1}\right)\right| \\
& \leq 2 \psi\left(2^{-(q+1)} Q\right) \leq 2 \psi\left(2^{-q} Q\right)
\end{aligned}
$$

so it is easy to see

$$
\left|\Delta_{i} \Delta_{j} F\right| \leq 2 \varrho\left[\varphi\left(2^{-p} P\right)\right] \sigma\left[\psi\left(2^{-q} Q\right)\right]
$$

for some increasing functions $\varrho, \sigma$ satisfying $\varrho(u) \sigma(u)=u$.

For the function $G$, let $M$ be its total $\Phi_{1}, \Psi_{1}$-variation, then

$$
\sum_{j=1}^{2^{q}} \Psi_{1}\left(\sum_{i=1}^{2^{p}} \Phi_{1}\left(\left|\Delta_{i} \Delta_{j} G\right|\right)\right) \leq M
$$

It is trivial to see that,

$$
2^{-q} \sum_{j=1}^{2^{q}} \Psi_{1}\left(\sum_{i=1}^{2^{p}} \Phi_{1}\left(\left|\Delta_{i} \Delta_{j} G\right|\right)\right) \leq 2^{-q} M
$$

As $\Psi_{1}$ is convex, so

$$
2^{-q} \sum_{j=1}^{2^{q}} \Psi_{1}\left(\sum_{i=1}^{2^{p}} \Phi_{1}\left(\left|\Delta_{i} \Delta_{j} G\right|\right)\right) \geq \Psi_{1}\left(2^{-q} \sum_{j=1}^{2^{q}} \sum_{i=1}^{2^{p}} \Phi_{1}\left(\left|\Delta_{i} \Delta_{j} G\right|\right)\right) .
$$

It turns out from (3.11) and (3.12) that

$$
\Psi_{1}\left(2^{-q} \sum_{j=1}^{2^{q}} \sum_{i=1}^{2^{p}} \Phi_{1}\left(\left|\Delta_{i} \Delta_{j} G\right|\right)\right) \leq 2^{-q} M
$$

This leads to

$$
2^{-q} \sum_{j=1}^{2^{q}} \sum_{i=1}^{2^{p}} \Phi_{1}\left(\left|\Delta_{i} \Delta_{j} G\right|\right) \leq \psi_{1}\left(2^{-q} M\right)
$$


This is equivalent to

$$
2^{-p} 2^{-q} \sum_{j=1}^{2^{q}} \sum_{i=1}^{2^{p}} \Phi_{1}\left(\left|\Delta_{i} \Delta_{j} G\right|\right) \leq 2^{-p} \psi_{1}\left(2^{-q} M\right) .
$$

But, by the convexity of $\Phi_{1}$, we have

$$
2^{-p} 2^{-q} \sum_{j=1}^{2^{q}} \sum_{i=1}^{2^{p}} \Phi_{1}\left(\left|\Delta_{i} \Delta_{j} G\right|\right) \geq \Phi_{1}\left(2^{-p} 2^{-q} \sum_{j=1}^{2^{q}} \sum_{i=1}^{2^{p}}\left|\Delta_{i} \Delta_{j} G\right|\right) .
$$

So it follows from (3.15) and (3.16) that

$$
\Phi_{1}\left(2^{-p} 2^{-q} \sum_{j=1}^{2^{q}} \sum_{i=1}^{2^{p}}\left|\Delta_{i} \Delta_{j} G\right|\right) \leq 2^{-p} \psi_{1}\left(2^{-q} M\right) .
$$

Therefore,

$$
2^{-p} 2^{-q} \sum_{j=1}^{2^{q}} \sum_{i=1}^{2^{p}}\left|\Delta_{i} \Delta_{j} G\right| \leq \varphi_{1}\left(2^{-p} \psi_{1}\left(2^{-q} M\right)\right) .
$$

So

$$
\sum_{j=1}^{2^{q}} \sum_{i=1}^{2^{p}}\left|\Delta_{i} \Delta_{j} G\right| \leq 2^{p+q} \varphi_{1}\left(2^{-p} \psi_{1}\left(2^{-q} M\right)\right)
$$

By the same method, one can see that

$$
\sum_{j=1,3,5, \cdots 2^{q+1}-1} \sum_{i=1,3,5, \cdots, 2^{p+1}-1}\left|\Delta_{i+1} \Delta_{j+1} G\right| \leq 2^{p+q} \varphi_{1}\left(2^{-p} \psi_{1}\left(2^{-q} M\right)\right) .(3.20)
$$

Therefore, it follows from (3.9), (3.10) and (3.20) that there exists $K>0$ such that

$$
\begin{aligned}
& \left|S\left(E_{p+1}, E_{q+1}^{\prime}\right)-S\left(E_{p+1}, E_{q}^{\prime}\right)-S\left(E_{p}, E_{q+1}^{\prime}\right)+S\left(E_{p}, E_{q}^{\prime}\right)\right| \\
\leq & K 2^{p+q} \varphi_{1}\left(2^{-p} \psi_{1}\left(2^{-q} M\right)\right) \varrho\left[\varphi\left(P 2^{-p}\right)\right] \sigma\left[\psi\left(Q 2^{-q}\right)\right] .
\end{aligned}
$$

Step 2: Let's prove that

$$
\lim _{p, q \rightarrow \infty} S\left(E+E_{p}, E^{\prime}+E_{q}^{\prime}\right)-S\left(E_{p}, E_{q}^{\prime}\right)=0 .
$$

Denoting $x_{l}, l=0,1, \cdots, L\left(y_{n}, n=0,1, \cdots, L^{\prime}\right)$ the distinct points of $E_{p}\left(E_{q}^{\prime}\right)$ in increasing order, and by $x_{l-1, i}, i=0,1, \cdots, M_{l}\left(y_{n-1, j}, j=\right.$ $\left.0,1, \cdots, M_{n}^{\prime}\right)$ those of $E+E_{p}\left(E^{\prime}+E_{q}^{\prime}\right)$ lying in the interval $x_{l-1} \leq x \leq x_{l}$ 
$\left(y_{n-1} \leq y \leq y_{n}\right)$ with $x_{l-1,0}=x_{l-1}, x_{l-1, M_{l}}=x_{l}\left(y_{n-1,0}=y_{n-1}, y_{n-1, M_{n}^{\prime}}=\right.$ $\left.y_{n}\right)$, so

$$
\begin{aligned}
& S\left(E+E_{p}, E^{\prime}+E_{q}^{\prime}\right)-S\left(E_{p}, E_{q}^{\prime}\right) \\
= & \left(S\left(E+E_{p}, E^{\prime}+E_{q}^{\prime}\right)-S\left(E+E_{p}, E_{q}^{\prime}\right)\right)+\left(S\left(E+E_{p}, E_{q}^{\prime}\right)-S\left(E_{p}, E_{q}^{\prime}\right)\right) \\
= & \sum_{l=1}^{L} \sum_{i=1}^{M_{l}} \sum_{n=1}^{L^{\prime}} \sum_{j=1}^{M_{n}^{\prime}}\left\{\left[F\left(x_{l-1, i-1}, y_{n-1, j-1}\right)-F\left(x_{l-1, i-1}, y_{n-1}\right)\right]\right. \\
& \left.\quad+\left[F\left(x_{l-1, i-1}, y_{n-1}\right)-F\left(x_{l-1}, y_{n-1}\right)\right]\right\}\left\{G\left(x_{l-1, i}, y_{n-1, j}\right)\right. \\
& \left.\quad-G\left(x_{l-1, i-1}, y_{n-1, j}\right)-G\left(x_{l-1, i}, y_{n-1, j-1}\right)+G\left(x_{l-1, i-1}, y_{n-1, j-1}\right)\right\} \\
\leq & 4 N_{1} N_{2}\left[\psi\left(2^{-q} Q\right)+\varphi\left(2^{-p} P\right)\right] \cdot \max |G| \\
\rightarrow & 0, \text { as } p, q \rightarrow \infty .
\end{aligned}
$$

Here $N_{1}, N_{2}$ denote the number of points of $E+E_{0}, E^{\prime}+E_{0}^{\prime}$, respectively.

Step 3: Let $F(x, y)$ vanish for $x=x^{\prime}$ identically in $y$, and for $y=y^{\prime}$ identically in $x$, so

$$
\begin{aligned}
& F_{E_{0}, E^{\prime}}(x, y)=F\left(x^{\prime}, y\right)=0, \quad F_{E, E_{0}^{\prime}}(x, y)=F\left(x, y^{\prime}\right)=0, \\
& F_{E_{0}, E_{0}^{\prime}}(x, y)=F\left(x^{\prime}, y^{\prime}\right)=0 .
\end{aligned}
$$

If this is so, and also note that $S\left(E, E^{\prime}\right)=S_{F_{E, E^{\prime}}}\left(E+E_{p}, E^{\prime}+E_{q}^{\prime}\right)$, then from Step 2, Step 1 and (3.5),

$$
\begin{aligned}
&\left|S\left(E, E^{\prime}\right)\right| \\
&=\left|S\left(E, E^{\prime}\right)-S\left(E_{0}, E^{\prime}\right)-S\left(E, E_{0}^{\prime}\right)+S\left(E_{0}, E_{0}^{\prime}\right)\right| \\
&= \mid \lim _{p, q \rightarrow \infty}\left[S_{F_{E, E^{\prime}}}\left(E+E_{p}, E^{\prime}+E_{q}^{\prime}\right)-S_{F_{E, E^{\prime}}}\left(E_{p}, E_{q}^{\prime}\right)+S_{F_{E, E^{\prime}}}\left(E_{p}, E_{q}^{\prime}\right)\right. \\
&\left.\quad-S_{F_{E, E^{\prime}}}\left(E_{0}, E_{q}^{\prime}\right)-S_{F_{E, E^{\prime}}}\left(E_{p}, E_{0}^{\prime}\right)+S_{F_{E, E^{\prime}}}\left(E_{0}, E_{0}^{\prime}\right)\right] \mid \\
&= \quad \lim _{p, q \rightarrow \infty}\left[S_{F_{E, E^{\prime}}}\left(E_{p}, E_{q}^{\prime}\right)-S_{F_{E, E^{\prime}}}\left(E_{0}, E_{q}^{\prime}\right)-S_{F_{E, E^{\prime}}}\left(E_{p}, E_{0}^{\prime}\right)\right. \\
&= \mid \sum_{p, q=0}^{\infty}\left[S_{F_{E, E^{\prime}}}\left(E_{p+1}, E_{q+1}^{\prime}\right)-S_{F_{E, E^{\prime}}}\left(E_{p+1}, E_{q}^{\prime}\right)\right] \\
& \leq\left.\sum_{p, q=0}^{\infty} K 2_{F_{E, E^{\prime}}}\left(E_{p}, E_{q+1}^{\prime}\right)+S_{F_{E, E^{\prime}}}\left(E_{p}, E_{q}^{\prime}\right)\right] \mid \\
& \leq\left.4 K\left(P 2^{-p}\right)\right] \sigma\left[\psi\left(Q 2^{-q}\right)\right] \varphi_{1}\left(2^{-p} \psi_{1}\left(2^{-q} M\right)\right) \\
& \sum_{m, n=1}^{\infty} \varrho\left[\varphi\left(\frac{P}{n}\right)\right] \sigma\left[\psi\left(\frac{Q}{m}\right)\right] \varphi_{1}\left(\frac{1}{n} \psi_{1}\left(\frac{1}{m} M\right)\right) .
\end{aligned}
$$


Let $F_{x^{\prime}, y^{\prime}}(x, y):=F(x, y)-F\left(x^{\prime}, y\right)-F\left(x, y^{\prime}\right)+F\left(x^{\prime}, y^{\prime}\right)$ and replace $F(x, y)$ by $F_{x^{\prime}, y^{\prime}}(x, y)$ for $x^{\prime} \leq x \leq x^{\prime \prime}, y^{\prime} \leq y \leq y^{\prime \prime}$. This alteration doesn't affect double difference of $F$. Therefore we may suppose that $F(x, y)$ vanish identically on the lines $x=x^{\prime}$ and $y=y^{\prime}$ as above.

Step 4: We determine a set of finite points $H_{v} \times H_{v^{\prime}}:=\left\{x^{\prime}=x_{0}<x_{1}<\right.$ $\left.\cdots<x_{L}=x^{\prime \prime}, y^{\prime}=y_{0}<y_{1}<\cdots<y_{L^{\prime}}=y^{\prime \prime}\right\}$, where $L \leq 2 \cdot 2^{v}, L^{\prime} \leq 2 \cdot 2^{v^{\prime}}$, such that in the rectangle $\left[x_{l-1}+\delta, x_{l}-\delta\right] \times\left[y_{k-1}+\delta, y_{k}-\delta\right],\left|\Delta_{i} \Delta_{j} G\right|<\varepsilon\left(v, v^{\prime}\right)$ for any $0<\delta \leq \frac{1}{4} \min \left\{\min _{1 \leq l \leq L}\left\{x_{l}-x_{l-1}\right\}, \min _{1 \leq l \leq L^{\prime}}\left\{y_{l}-y_{l-1}\right\}\right\}$. Moreover, in the interval $\left[x^{\prime}, x^{\prime \prime}\right] \times\left[y_{k-1}+\delta, y_{k}-\delta\right]$, the total $\Psi$-variation of $F$ in $y$ is at most $Q \cdot 2^{-v^{\prime}}$, the total $\Phi_{1}, \Psi_{1}$ - variation of $G$ is at most $M \cdot 2^{-v^{\prime}}$; in the interval $\left[x_{l-1}+\delta, x_{l}-\delta\right] \times\left[y^{\prime}, y^{\prime \prime}\right]$, the total $\Phi$-variation of $F$ in $x$ is at most $P \cdot 2^{-v}$ and the total $\Phi_{1}$-variation of $G$ in $x$ for the given partition of $H_{v}^{\prime}$ of $\left[y^{\prime}, y^{\prime \prime}\right]$ is at most $2^{-v} L^{\prime} \psi_{1}\left(\frac{1}{L^{\prime}} M\right)$. Here, the first and the third statements are obvious, the second statement follows from the finite large jump condition. The last one can be seen by observing that $\sum_{j=1}^{L^{\prime}} \Psi_{1}\left(\sum_{i=1}^{L} \Phi_{1}\left(\left|\Delta_{i} \Delta_{j} G\right|\right)\right) \leq M$ is equivalent to $\sum_{j=1}^{L^{\prime}} \sum_{i=1}^{L} \Phi_{1}\left(\left|\Delta_{i} \Delta_{j} G\right|\right) \leq L^{\prime} \psi_{1}\left(\frac{1}{L^{\prime}} M\right)$. More generally, for any partition $E=\left\{x_{1}^{\prime}, x_{2}^{\prime}, \cdots, x_{N}^{\prime}\right\}$, we have $\sum_{i=1}^{N} \sum_{j=1}^{L^{\prime}} \Phi_{1}\left(\left|\Delta_{i}^{\prime} \Delta_{j} G\right|\right) \leq L^{\prime} \psi_{1}\left(\frac{1}{L^{\prime}} M\right)$. Here $\Delta_{i}^{\prime} \Delta_{j} G$ is the double increment of $G$ on $\left(x_{i-1}^{\prime}, x_{i}^{\prime}\right) \times\left(y_{j-1}, y_{j}\right)$. We can make $E$ include $H_{v}$ among their points of divisions and denote $E_{l-1}=$ $\left\{x_{l-1,1}, x_{l-1,2}, \cdots, x_{l-1, N_{l-1}}\right\}$ all the points in $E$ falling into the interval $\left(x_{l-1}, x_{l}\right)(l=1,2, \cdots, L)$. We can certainly make

$$
\sum_{i=1}^{N_{l-1}} \sum_{j=1}^{L^{\prime}} \Phi_{1}\left(\left|\Delta_{l-1, i}^{\prime} \Delta_{j} G\right|\right) \leq 2^{-v} L^{\prime} \psi_{1}\left(\frac{1}{L^{\prime}} M\right),
$$

where $\Delta_{l-1, i}^{\prime} \Delta_{j} G$ is the double increment of $G$ on $\left(x_{l-1, i-1}^{\prime}, x_{l-1, i}^{\prime}\right) \times\left(y_{j-1}, y_{j}\right)$. In fact $E_{l-1}$ can be any partition of $\left[x_{l-1}+\delta, x_{l}-\delta\right]$ for any sufficiently small $\delta>0$.

We need to prove that for any $\varepsilon>0$,

$$
\left|S\left(\tilde{D}, \tilde{D}^{\prime}\right)-S\left(D, D^{\prime}\right)\right|<\varepsilon,
$$

as long as $\tilde{D} \times \tilde{D}^{\prime}$, and $D \times D^{\prime}$ include $H_{v} \times H_{v^{\prime}}$. Observe that

$$
\begin{aligned}
& \left|S\left(\tilde{D}, \tilde{D}^{\prime}\right)-S\left(D, D^{\prime}\right)\right| \\
\leq & \left|S\left(\tilde{D}, \tilde{D}^{\prime}\right)-S\left(\tilde{D}, D^{\prime}\right)\right|+\left|S\left(\tilde{D}, D^{\prime}\right)-S\left(D, D^{\prime}\right)\right| \\
\leq & \left|\int_{x^{\prime}}^{x^{\prime \prime}} \int_{y^{\prime}}^{y^{\prime \prime}}\left(F_{\tilde{D}, \tilde{D^{\prime}}}-F_{\tilde{D}, D^{\prime}}\right) d_{x, y} G(x, y)\right| \\
& +\left|\int_{x^{\prime}}^{x^{\prime \prime}} \int_{y^{\prime}}^{y^{\prime \prime}}\left(F_{\tilde{D}, D^{\prime}}-F_{D, D^{\prime}}\right) d_{x, y} G(x, y)\right| .
\end{aligned}
$$

First, since $F_{\tilde{D}, \tilde{D}^{\prime}}-F_{\tilde{D}, D^{\prime}}$ vanishes identically in $x$, when $y=y_{k-1}$, from Step 3 and (3.5), (3.6), we obtain for any sufficiently small $\delta>0$, 


$$
\begin{aligned}
& \left|S\left(\tilde{D}, \tilde{D}^{\prime}\right)-S\left(\tilde{D}, D^{\prime}\right)\right| \\
\leq & \sum_{k=1}^{L^{\prime}}\left|\int_{x^{\prime}}^{x^{\prime \prime}} \int_{y_{k-1}+\delta}^{y_{k}-\delta}\left(F_{\tilde{D}, \tilde{D^{\prime}}}-F_{\tilde{D}, D^{\prime}}\right) d_{x, y} G(x, y)\right| \\
\leq & \sum_{k=1}^{L^{\prime}} 4 K \sum_{m, n=1}^{\infty} \varrho\left[\varphi\left(\frac{P}{n}\right)\right] \sigma\left[\psi\left(\frac{2^{-v^{\prime}} Q}{m}\right)\right] \varphi_{1}\left[\frac{1}{n} \psi_{1}\left(\frac{1}{m} 2^{-v^{\prime}} M\right)\right] \\
\leq & \sum_{k=1}^{L^{\prime}} 4 K \sum_{n=1}^{\infty} \sum_{q=0}^{\infty} \varrho\left[\varphi\left(\frac{P}{n}\right)\right] 2^{q} \sigma\left[\psi\left(2^{-\left(v^{\prime}+q\right)} Q\right)\right] \varphi_{1}\left[\frac{1}{n} \psi_{1}\left(2^{-\left(v^{\prime}+q\right)} M\right)\right] \\
= & L^{\prime} 4 K \sum_{n=1}^{\infty} \sum_{q=v^{\prime}}^{\infty} \varrho\left[\varphi\left(\frac{P}{n}\right)\right] 2^{-v^{\prime}+q} \sigma\left[\psi\left(2^{-q} Q\right)\right] \varphi_{1}\left[\frac{1}{n} \psi_{1}\left(2^{-q} M\right)\right] \\
\leq & 16 K \sum_{n=1}^{\infty} \sum_{m=2^{v^{\prime}-1}+1}^{\infty} \varrho\left[\varphi\left(\frac{P}{n}\right)\right] \sigma\left[\psi\left(\frac{Q}{m}\right)\right] \varphi_{1}\left[\frac{1}{n} \psi_{1}\left(\frac{1}{m} M\right)\right] \\
\leq & \varepsilon_{v^{\prime}},
\end{aligned}
$$

where $\varepsilon_{v^{\prime}} \rightarrow 0$, as $v^{\prime} \rightarrow \infty$.

Second, since $F_{\tilde{D}, D^{\prime}}-F_{D, D^{\prime}}$ vanishes identically in $y$, when $x=x_{l-1}$. From the discussion above and (3.23), we know for any partition $E_{p}=$ $\left\{x_{1}, x_{2}, \cdots, x_{2^{p}}\right\}$ of $\left[x_{l-1}+\delta, x_{l}-\delta\right]$, and any partition $E_{q^{\prime}}=\left\{y_{1}, y_{2}, \cdots, y_{2^{q}}\right\}$ of $\left[y^{\prime}, y^{\prime \prime}\right],(3.14)$ bacomes

$$
2^{-q} \sum_{j=1}^{2^{q}} \sum_{i=1}^{2^{p}} \Phi_{1}\left(\left|\Delta_{i} \Delta_{j} G\right|\right) \leq 2^{-v} \psi_{1}\left(2^{-q} M\right) .
$$

So from Step 3 and (3.5), (3.6),

$$
\begin{aligned}
& \left|\int_{x_{l-1}+\delta}^{x_{l}-\delta} \int_{y^{\prime}}^{y^{\prime \prime}}\left(F_{\tilde{D}, D^{\prime}}-F_{D, D^{\prime}}\right) d_{x, y} G(x, y)\right| \\
\leq & 4 K \sum_{m, n=1}^{\infty} \varrho\left[\varphi\left(\frac{2^{-v} P}{n}\right)\right] \sigma\left[\psi\left(\frac{Q}{m}\right)\right] \varphi_{1}\left[\frac{2^{-v}}{n} \psi_{1}\left(\frac{1}{m} M\right)\right] \\
\leq & 4 K \sum_{m=1}^{\infty} \sum_{q=0}^{\infty} 2^{q} \varrho\left[\varphi\left(2^{-(v+q)} P\right)\right] \sigma\left[\psi\left(\frac{Q}{m}\right)\right] \varphi_{1}\left[2^{-(v+q)} \psi_{1}\left(\frac{1}{m} M\right)\right] \\
= & 4 K 2^{-v} \sum_{m=1}^{\infty} \sum_{q=v}^{\infty} 2^{q} \varrho\left[\varphi\left(2^{-q} P\right)\right] \sigma\left[\psi\left(\frac{Q}{m}\right)\right] \varphi_{1}\left[2^{-q} \psi_{1}\left(\frac{1}{m} M\right)\right] \\
\leq & 8 K 2^{-v} \sum_{m=1}^{\infty} \sum_{n=2^{v-1}+1}^{\infty} \varrho\left[\varphi\left(\frac{P}{n}\right)\right] \sigma\left[\psi\left(\frac{Q}{m}\right)\right] \varphi_{1}\left[\frac{1}{n} \psi_{1}\left(\frac{1}{m} M\right)\right] .
\end{aligned}
$$

Now it turns out that 


$$
\begin{aligned}
& \left|S\left(\tilde{D}, D^{\prime}\right)-S\left(D, D^{\prime}\right)\right| \\
\leq & \sum_{l=1}^{L}\left|\int_{x_{l-1}+\delta}^{x_{l}-\delta} \int_{y^{\prime}}^{y^{\prime \prime}}\left(F_{\tilde{D}, D^{\prime}}-F_{D, D^{\prime}}\right) d_{x, y} G(x, y)\right| \\
\leq & 16 K \sum_{m=1}^{\infty} \sum_{n=2^{v-1}+1}^{\infty} \varrho\left[\varphi\left(\frac{P}{n}\right)\right] \sigma\left[\psi\left(\frac{Q}{m}\right)\right] \varphi_{1}\left[\frac{1}{n} \psi_{1}\left(\frac{1}{m} M\right)\right] \\
\leq & \varepsilon_{v},
\end{aligned}
$$

where $\varepsilon_{v} \rightarrow 0$, as $v \rightarrow \infty$.

Thus we can get (3.24) from (3.25), (3.26) and (3.28), as $v, v^{\prime} \rightarrow \infty$, which means $S\left(D, D^{\prime}\right)$ is a Cauchy sequence, so $\lim _{m\left(D \times D^{\prime}\right) \rightarrow 0} S\left(D, D^{\prime}\right)$ exists. In the following, we show the limit is unique. For this, let $D_{1} \times D_{1}^{\prime}, D_{2} \times D_{2}^{\prime}$ be arbitrary two partitions of $\left[x^{\prime}, x^{\prime \prime}\right] \times\left[y^{\prime}, y^{\prime \prime}\right]$ including $H \times H^{\prime}$. From the above we know,

$$
\begin{aligned}
& \left|S\left(D_{1} \cup D_{2}, D_{1}^{\prime} \cup D_{2}^{\prime}\right)-S\left(D_{1}, D_{1}^{\prime}\right)\right| \rightarrow 0 \text {, as } m\left(D_{1} \times D_{1}^{\prime}\right) \rightarrow 0, \\
& \left|S\left(D_{1} \cup D_{2}, D_{1}^{\prime} \cup D_{2}^{\prime}\right)-S\left(D_{2}, D_{2}^{\prime}\right)\right| \rightarrow 0 \text {, as } m\left(D_{2} \times D_{2}^{\prime}\right) \rightarrow 0 .
\end{aligned}
$$

Therefore,

$$
\begin{aligned}
& \lim _{m\left(D_{1} \times D_{1}^{\prime}\right) \rightarrow 0} S\left(D_{1}, D_{1}^{\prime}\right)=\lim _{m\left(D_{2} \times D_{2}^{\prime}\right) \rightarrow 0} S\left(D_{2}, D_{2}^{\prime}\right) \\
& =\lim _{m\left(D_{1} \times D_{1}^{\prime}\right), m\left(D_{2} \times D_{2}^{\prime}\right) \rightarrow 0} S\left(D_{1} \cup D_{2}, D_{1}^{\prime} \cup D_{2}^{\prime}\right),
\end{aligned}
$$

that is to say, $\lim _{m\left(D \times D^{\prime}\right) \rightarrow 0} S\left(D, D^{\prime}\right)$ is unique, and we define it as $\int_{x^{\prime}}^{x^{\prime \prime}} \int_{y^{\prime}}^{y^{\prime \prime}} F(x, y) d_{x, y} G(x, y)$. So we proved our theorem.

In the following we say an integral is well defined which is in the sense of Theorem 3.1. The following convergence theorem plays an important role in establishing Itô's formula:

Theorem 3.2 Assume there exist convex functions $\Phi, \Psi, \Phi_{1}, \Psi_{1}$ such that $F_{k}(x, y)$ and $F(x, y)$ are continuous functions of bounded $\Phi$-variation in $x$ uniformly in $y$ and of bounded $\Psi$-variation in $y$ uniformly in $x$ and for $F_{k}$ uniformly in $k, G(x, y)$ and $G_{k}(x, y)$ are of bounded $\Phi_{1}, \Psi_{1}$-variation in $(x, y)$ uniformly in $k$ and satisfies the finite large jump condition. If there exist $\varrho_{i}$ and $\sigma_{i}$ subject to $\varrho_{i}(u) \sigma_{i}(u)=u, i=1,2$, and a small positive number $\delta>0$ such that

$$
\begin{aligned}
& \sum_{m, n} \varrho_{1}\left[\varphi\left(\frac{1}{n}\right)\right] \sigma_{1}\left[\psi\left(\frac{1}{m}\right)\right] \varphi_{1}^{\frac{1}{1+\delta}}\left[\frac{1}{n} \psi_{1}\left(\frac{1}{m}\right)\right] \\
& +\sum_{m, n} \varrho_{2}\left[\varphi\left(\left(\frac{1}{n}\right)^{\frac{1}{1+\delta}}\right)\right] \sigma_{2}\left[\psi\left(\frac{1}{m}\right)\right] \varphi_{1}\left[\frac{1}{n} \psi_{1}\left(\frac{1}{m}\right)\right]<\infty,
\end{aligned}
$$


or

$$
\begin{aligned}
& \sum_{m, n} \varrho_{1}\left[\varphi\left(\frac{1}{n}\right)\right] \sigma_{1}\left[\psi\left(\frac{1}{m}\right)\right] \varphi_{1}^{\frac{1}{1+\delta}}\left[\frac{1}{n} \psi_{1}\left(\frac{1}{m}\right)\right] \\
& +\sum_{m, n} \varrho_{2}\left[\varphi\left(\frac{1}{n}\right)\right] \sigma_{2}\left[\psi\left(\left(\frac{1}{m}\right)^{\frac{1}{1+\delta}}\right)\right] \varphi_{1}\left[\frac{1}{n} \psi_{1}\left(\frac{1}{m}\right)\right]<\infty,
\end{aligned}
$$

and let $F_{k}(x, y) \rightarrow F(x, y), G_{k}(x, y) \rightarrow G(x, y)$ as $k \rightarrow \infty$ uniformly in $(x, y)$. Then we have

$$
\int_{x^{\prime}}^{x^{\prime \prime}} \int_{y^{\prime}}^{y^{\prime \prime}} F_{k}(x, y) d_{x, y} G_{k}(x, y) \rightarrow \int_{x^{\prime}}^{x^{\prime \prime}} \int_{y^{\prime}}^{y^{\prime \prime}} F(x, y) d_{x, y} G(x, y),
$$

when $k \rightarrow \infty$.

Proof: First note that from Theorem 3.1 under the above assumptions, the integral $\int_{x^{\prime}}^{x^{\prime \prime}} \int_{y^{\prime}}^{y^{\prime \prime}} F_{k}(x, y) d_{x, y} G_{k}(x, y)$ and $\int_{x^{\prime}}^{x^{\prime \prime}} \int_{y^{\prime}}^{y^{\prime \prime}} F(x, y) d_{x, y} G(x, y)$ are all well defined. It's easy to see that

$$
\begin{aligned}
& \frac{1}{2}\left(\int_{x^{\prime}}^{x^{\prime \prime}} \int_{y^{\prime}}^{y^{\prime \prime}} F_{k}(x, y) d_{x, y} G_{k}(x, y)-\int_{x^{\prime}}^{x^{\prime \prime}} \int_{y^{\prime}}^{y^{\prime \prime}} F(x, y) d_{x, y} G(x, y)\right) \\
= & \int_{x^{\prime}}^{x^{\prime \prime}} \int_{y^{\prime}}^{y^{\prime \prime}} F_{k}(x, y) d_{x, y} \frac{1}{2}\left(G_{k}(x, y)-G(x, y)\right) \\
& +\int_{x^{\prime}}^{x^{\prime \prime}} \int_{y^{\prime}}^{y^{\prime \prime}} \frac{1}{2}\left(F_{k}(x, y)-F(x, y)\right) d_{x, y} G(x, y) .
\end{aligned}
$$

We study $\frac{1}{2}$ of the integral only for convenience in what follows. First consider the integral $\int_{x^{\prime}}^{x^{\prime \prime}} \int_{y^{\prime}}^{y^{\prime \prime}} F_{k}(x, y) d_{x, y}\left(G_{k}(x, y)-G(x, y)\right)$. Note there exist constant $P_{1}, Q_{1}, M_{1}, M_{2}>0$, which are independent of $k$ such that for any partition $E \times E^{\prime}$ defined before

$$
\begin{aligned}
& \sum_{i=1}^{N} \Phi\left(\left|F_{k}\left(x_{i}, y\right)-F_{k}\left(x_{i-1}, y\right)\right|\right) \leq P_{1}, \quad \text { for any } y \in\left[y^{\prime}, y^{\prime \prime}\right] \\
& \sum_{j=1}^{N^{\prime}} \Psi\left(\left|F_{k}\left(x, y_{j}\right)-F_{k}\left(x, y_{j-1}\right)\right|\right) \leq Q_{1}, \quad \text { for any } x \in\left[x^{\prime}, x^{\prime \prime}\right] \\
& \sum_{j=1}^{N^{\prime}} \Psi_{1}\left(\sum_{i=1}^{N} \Phi_{1}\left(\left|\Delta_{i} \Delta_{j} G_{k}\right|\right)\right) \leq M_{1} \\
& \sum_{j=1}^{N^{\prime}} \Psi_{1}\left(\sum_{i=1}^{N} \Phi_{1}\left(\left|\Delta_{i} \Delta_{j} G\right|\right)\right) \leq M_{2}
\end{aligned}
$$

For the small $\delta>0$ given in condition (3.29), from the convexity of $\Phi_{1}$ and $\Psi_{1}$ and $G_{k} \rightarrow G$ when $k \rightarrow \infty$, we have 


$$
\begin{aligned}
& \sum_{j=1}^{N^{\prime}} \Psi_{1}\left(\sum_{i=1}^{N} \Phi_{1}\left(\left|\Delta_{i} \Delta_{j} \frac{1}{2}\left(G_{k}-G\right)\right|^{1+\delta}\right)\right) \\
= & \sum_{j=1}^{N^{\prime}} \Psi_{1}\left(\sum_{i=1}^{N} \Phi_{1}\left(\left|\Delta_{i} \Delta_{j} \frac{1}{2}\left(G_{k}-G\right)\right|^{\delta} \cdot\left|\Delta_{i} \Delta_{j} \frac{1}{2}\left(G_{k}-G\right)\right|\right)\right) \\
\leq & \sum_{j=1}^{N^{\prime}} \Psi_{1}\left(\sum_{i=1}^{N}\left|\Delta_{i} \Delta_{j} \frac{1}{2}\left(G_{k}-G\right)\right|^{\delta} \Phi_{1}\left(\left|\Delta_{i} \Delta_{j} \frac{1}{2}\left(G_{k}-G\right)\right|\right)\right) \\
\leq & \sum_{j=1}^{N^{\prime}} \Psi_{1}\left(\max _{i}\left|\Delta_{i} \Delta_{j} \frac{1}{2}\left(G_{k}-G\right)\right|^{\delta} \sum_{i=1}^{N} \Phi_{1}\left(\left|\Delta_{i} \Delta_{j} \frac{1}{2}\left(G_{k}-G\right)\right|\right)\right) \\
\leq & \sum_{j=1}^{N^{\prime}} \max _{i}\left|\Delta_{i} \Delta_{j} \frac{1}{2}\left(G_{k}-G\right)\right|^{\delta} \Psi_{1}\left(\sum_{i=1}^{N} \Phi_{1}\left(\frac{1}{2}\left|\Delta_{i} \Delta_{j} G_{k}\right|+\frac{1}{2}\left|\Delta_{i} \Delta_{j} G\right|\right)\right) \\
\leq & \max _{i, j}\left|\Delta_{i} \Delta_{j}\left(G_{k}-G\right)\right|^{\delta} \sum_{j=1}^{N^{\prime}} \Psi_{1}\left(\sum_{i=1}^{N}\left(\frac{1}{2} \Phi_{1}\left(\left|\Delta_{i} \Delta_{j} G_{k}\right|\right)+\frac{1}{2} \Phi_{1}\left(\left|\Delta_{i} \Delta_{j} G\right|\right)\right)\right) \\
\leq & \varepsilon_{1}(k) M
\end{aligned}
$$

where $\varepsilon_{1}(k) \rightarrow 0$ as $k \rightarrow \infty$, and $M$ is a constant independent of $k$. If we define

$$
S\left(E, E^{\prime}\right)=\sum_{i=1}^{N} \sum_{j=1}^{N^{\prime}} F_{k}\left(x_{i-1}, y_{j-1}\right)\left(\Delta_{i} \Delta_{j}\left(G_{k}-G\right)\right),
$$

and similar to (3.22), by dominated convergence theorem to the infinite series,

$\left|S\left(E, E^{\prime}\right)\right| \leq 4 K \sum_{m, n} \varrho_{1}\left[\varphi\left(\frac{P_{1}}{n}\right)\right] \sigma_{1}\left[\psi\left(\frac{Q_{1}}{m}\right)\right] \varphi_{1}^{\frac{1}{1+\delta}}\left[\frac{1}{n} \psi_{1}\left(\frac{2 \varepsilon_{1}(k)}{m} M\right)\right] \rightarrow 0$, as $k \rightarrow \infty$,

as the series $\sum_{m, n} \varrho_{1}\left[\varphi\left(\frac{1}{n}\right)\right] \sigma_{1}\left[\psi\left(\frac{1}{m}\right)\right] \varphi_{1}^{\frac{1}{1+\delta}}\left[\frac{1}{n} \psi_{1}\left(\frac{1}{m}\right)\right]<\infty$. This implies as $k \rightarrow$ $\infty$

$$
\lim _{k \rightarrow \infty} \int_{x^{\prime}}^{x^{\prime \prime}} \int_{y^{\prime}}^{y^{\prime \prime}} F_{k}(x, y) d_{x, y}\left(G_{k}(x, y)-G(x, y)\right)=0
$$

For the second integral $\int_{x^{\prime}}^{x^{\prime \prime}} \int_{y^{\prime}}^{y^{\prime \prime}}\left(F_{k}(x, y)-F(x, y)\right) d_{x, y} G(x, y)$, we can use a similar method to prove

$$
\lim _{k \rightarrow \infty} \int_{x^{\prime}}^{x^{\prime \prime}} \int_{y^{\prime}}^{y^{\prime \prime}}\left(F_{k}(x, y)-F(x, y)\right) d_{x, y} G(x, y)=0 .
$$

For this, we note from the assumption there is a $\delta>0$ such that, 


$$
\begin{aligned}
& \sum_{i=1}^{N} \Phi^{1+\delta}\left(\left|\frac{1}{2}\left(F_{k}-F\right)\left(x_{i}, y\right)-\frac{1}{2}\left(F_{k}-F\right)\left(x_{i-1}, y\right)\right|\right) \\
\leq & \max _{i} \Phi^{\delta}\left(\left|\frac{1}{2}\left(F_{k}-F\right)\left(x_{i}, y\right)-\frac{1}{2}\left(F_{k}-F\right)\left(x_{i-1}, y\right)\right|\right) . \\
& \sum_{i=1}^{N} \frac{1}{2} \Phi\left(\left(\left|F_{k}\left(x_{i}, y\right)-F_{k}\left(x_{i-1}, y\right)\right|\right)+\Phi\left(\left|F_{k}\left(x_{i}, y\right)-F_{k}\left(x_{i-1}, y\right)\right|\right)\right) \\
\leq & \varepsilon_{2}(k) M^{\prime},
\end{aligned}
$$

where $\varepsilon_{2}(k) \rightarrow 0$, as $k \rightarrow \infty$, and $M^{\prime}$ is a constant independent of $k$. So under the assumption $\sum_{m, n} \varrho_{2}\left[\varphi\left(\left(\frac{1}{n}\right)^{\frac{1}{1+\delta}}\right)\right] \sigma_{2}\left[\psi\left(\frac{1}{m}\right)\right] \varphi_{1}\left[\frac{1}{n} \psi_{1}\left(\frac{1}{m}\right)\right]<\infty$, we can prove (3.37) using the same argument in proving (3.36). Therefore under assumption (3.29), we prove the desired result. The proof is similar under the assumption (3.30).

Remark 3.1 From the proof we can easily see that under the condition that there exist two functions $\varrho$ and $\sigma$ subject to $\varrho(u) \sigma(u)=u$ and a small number $\delta>0$ such that

$$
\sum_{m, n} \varrho\left[\varphi\left(\frac{1}{n}\right)\right] \sigma\left[\psi\left(\frac{1}{m}\right)\right] \varphi_{1}^{\frac{1}{1+\delta}}\left[\frac{1}{n} \psi_{1}\left(\frac{1}{m}\right)\right]<\infty
$$

Then as $k \rightarrow \infty$,

$$
\int_{x^{\prime}}^{x^{\prime \prime}} \int_{y^{\prime}}^{y^{\prime \prime}} F(x, y) d_{x, y} G_{k}(x, y) \rightarrow \int_{x^{\prime}}^{x^{\prime \prime}} \int_{y^{\prime}}^{y^{\prime \prime}} F(x, y) d_{x, y} G(x, y) .
$$

Similarly, under the condition that there exist two functions $\varrho$ and $\sigma$ subject to $\varrho(u) \sigma(u)=u$ and a small number $\delta>0$ such that

$$
\sum_{m, n} \varrho\left[\varphi\left(\left(\frac{1}{n}\right)^{\frac{1}{1+\delta}}\right)\right] \sigma\left[\psi\left(\frac{1}{m}\right)\right] \varphi_{1}\left[\frac{1}{n} \psi_{1}\left(\frac{1}{m}\right)\right]<\infty
$$

or

$$
\sum_{m, n} \varrho\left[\varphi\left(\frac{1}{n}\right)\right] \sigma\left[\psi\left(\left(\frac{1}{m}\right)^{\frac{1}{1+\delta}}\right)\right] \varphi_{1}\left[\frac{1}{n} \psi_{1}\left(\frac{1}{m}\right)\right]<\infty
$$

Then as $k \rightarrow \infty$,

$$
\int_{x^{\prime}}^{x^{\prime \prime}} \int_{y^{\prime}}^{y^{\prime \prime}} F_{k}(x, y) d_{x, y} G(x, y) \rightarrow \int_{x^{\prime}}^{x^{\prime \prime}} \int_{y^{\prime}}^{y^{\prime \prime}} F(x, y) d_{x, y} G(x, y) .
$$

It is easy to see that in the definition of $\int_{x^{\prime}}^{x^{\prime \prime}} \int_{y^{\prime}}^{y^{\prime \prime}} F(x, y) d_{x, y} G(x, y)$, one can take $F\left(x_{i}, y_{j}\right)$ instead of $F\left(x_{i-1}, y_{j-1}\right)$ in (3.8). One can also 
prove the convergence of (3.8) in this case and denote the integral by $\int_{x^{\prime}}^{x^{\prime \prime}} \int_{y^{\prime}}^{y^{\prime \prime}} F(x, y) d_{x, y}^{*} G(x, y)$, the backward integral. In general, this should be different from $\int_{x^{\prime}}^{x^{\prime \prime}} \int_{y^{\prime}}^{y^{\prime \prime}} F(x, y) d_{x, y} G(x, y)$. But under slightly stronger conditions than those in Theorem 3.1, as in the one-parameter case, these two integrals equal. This result is proved in the following proposition.

Proposition 3.1 Assume there exist convex functions $\Phi, \Psi, \Phi_{1}, \Psi_{1}$ such that $F(x, y)$ is a continuous function of bounded $\Phi$-variation in $x$ uniformly in $y$ and of bounded $\Psi$-variation in $y$ uniformly in $x, G(x, y)$ is of bounded $\Phi_{1}, \Psi_{1}$-variation in $(x, y)$ and satifies the finite large jump condition. If there exist functions $\varrho$ and $\sigma$ subject to $\varrho(u) \sigma(u)=u$ and a small positive $\delta>0$ such that one of the following two conditions is satisfied

(i) $F(x, y)$ is continuous in $x$ and

$$
\sum_{m, n} \varrho\left[\varphi\left(\left(\frac{1}{n}\right)^{\frac{1}{1+\delta}}\right)\right] \sigma\left[\psi\left(\frac{1}{m}\right)\right] \varphi_{1}\left[\frac{1}{n} \psi_{1}\left(\frac{1}{m}\right)\right]<\infty,
$$

(ii) $F(x, y)$ is continuous in $y$ and

$$
\sum_{m, n} \varrho\left[\varphi\left(\frac{1}{n}\right)\right] \sigma\left[\psi\left(\left(\frac{1}{m}\right)^{\frac{1}{1+\delta}}\right)\right] \varphi\left[\frac{1}{n} \psi_{1}\left(\frac{1}{m}\right)\right]<\infty .
$$

Then

$$
\int_{x^{\prime}}^{x^{\prime \prime}} \int_{y^{\prime}}^{y^{\prime \prime}} F(x, y) d_{x, y} G(x, y)=\int_{x^{\prime}}^{x^{\prime \prime}} \int_{y^{\prime}}^{y^{\prime \prime}} F(x, y) d_{x, y}^{*} G(x, y) .
$$

Proof: We only prove the result when condition (i) is satisfied. Denote

$$
\begin{aligned}
& S\left(E, E^{\prime}\right)=\sum_{i=1}^{N} \sum_{j=1}^{N^{\prime}} F\left(x_{i-1}, y_{j-1}\right) \Delta_{i} \Delta_{j} G, \\
& S^{*}\left(E, E^{\prime}\right)=\sum_{i=1}^{N} \sum_{j=1}^{N^{\prime}} F\left(x_{i}, y_{j}\right) \Delta_{i} \Delta_{j} G .
\end{aligned}
$$

Here $E$ and $E^{\prime}$ are the same as before. Denote

$$
\tilde{F}_{\delta_{x_{i-1}}, \delta_{y_{j-1}}}\left(x_{i-1}, y_{j-1}\right)=F\left(x_{i-1}+\delta_{x_{i-1}}, y_{j-1}+\delta_{y_{j-1}}\right)-F\left(x_{i-1}, y_{j-1}\right) .
$$

Here $\delta_{x_{i-1}}=x_{i}-x_{i-1}, \delta_{y_{j-1}}=y_{j}-y_{j-1}$. Then

$$
S^{*}\left(E, E^{\prime}\right)-S\left(E, E^{\prime}\right)=2 \sum_{i=1}^{N} \sum_{j=1}^{N^{\prime}} \frac{1}{2} \tilde{F}_{\delta_{x_{i-1}}, \delta_{y_{j-1}}}\left(x_{i-1}, y_{j-1}\right) \Delta_{i} \Delta_{j} G .
$$

Note from the assumptions, there is a $\delta>0$ such that 


$$
\begin{aligned}
& \sum_{i=1}^{N} \Phi^{1+\delta}\left(\left|\frac{1}{2}\left[\tilde{F}_{\delta_{x_{i}}, \delta_{y_{j}-1}}\left(x_{i}, y_{j-1}\right)-\tilde{F}_{\delta_{x_{i-1}}, \delta_{y_{j-1}}}\left(x_{i-1}, y_{j-1}\right)\right]\right|\right) \\
\leq & \max _{i, j} \Phi^{\delta}\left(\frac{1}{2}\left|\left[F\left(x_{i+1}, y_{j}\right)-F\left(x_{i}, y_{j-1}\right)\right]-\left[F\left(x_{i}, y_{j}\right)-F\left(x_{i-1}, y_{j-1}\right)\right]\right|\right) \\
& \sum_{i=1}^{N} \Phi\left(\left|\frac{1}{2}\left[F\left(x_{i+1}, y_{j}\right)-F\left(x_{i}, y_{j}\right)\right]-\frac{1}{2}\left[F\left(x_{i}, y_{j-1}\right)-F\left(x_{i-1}, y_{j-1}\right)\right]\right|\right) \\
\leq & \max _{i, j} \Phi^{\delta}\left(\frac{1}{2}\left|\left[F\left(x_{i+1}, y_{j}\right)-F\left(x_{i}, y_{j}\right)\right]-\left[F\left(x_{i}, y_{j-1}\right)-F\left(x_{i-1}, y_{j-1}\right)\right]\right|\right) \\
& \sum_{i=1}^{N} \frac{1}{2}\left(\Phi\left(\left|F\left(x_{i+1}, y_{j}\right)-F\left(x_{i}, y_{j}\right)\right|\right)+\Phi\left(\left|F\left(x_{i}, y_{j-1}\right)-F\left(x_{i-1}, y_{j-1}\right)\right|\right)\right) \\
\leq & \varepsilon\left(E, E^{\prime}\right) P
\end{aligned}
$$

where $\varepsilon\left(E, E^{\prime}\right) \rightarrow 0$, when $m\left(E, E^{\prime}\right) \rightarrow 0$ and $P$ is a constant. Therefore following (3.22), we see that

$$
\begin{aligned}
& \left|S^{*}\left(E, E^{\prime}\right)-S\left(E, E^{\prime}\right)\right| \\
= & \left|\sum_{i=1}^{N} \sum_{j=1}^{N^{\prime}} \tilde{F}_{\delta_{x_{i-1}}, \delta_{y_{j-1}}}\left(x_{i-1}, y_{j-1}\right) \Delta_{i} \Delta_{j} G\right| \\
\leq & 8 K \sum_{m, n=1}^{\infty} \varrho\left[\varphi\left(\left(\frac{2 \varepsilon\left(E, E^{\prime}\right) P}{n}\right)^{\frac{1}{1+\delta}}\right)\right] \sigma\left[\psi\left(\frac{Q}{m}\right)\right] \varphi_{1}\left(\frac{1}{n} \psi_{1}\left(\frac{1}{m} M\right)\right) \\
\rightarrow & 0, \text { as } \varepsilon\left(E, E^{\prime}\right) \rightarrow 0 .
\end{aligned}
$$

Therefore

$$
S^{*}\left(E, E^{\prime}\right)-S\left(E, E^{\prime}\right) \rightarrow 0 \text { as } \varepsilon\left(E, E^{\prime}\right) \rightarrow 0 .
$$

That is to say,

$$
\int_{x^{\prime}}^{x^{\prime \prime}} \int_{y^{\prime}}^{y^{\prime \prime}} F(x, y) d_{x, y} G(x, y)=\int_{x^{\prime}}^{x^{\prime \prime}} \int_{y^{\prime}}^{y^{\prime \prime}} F(x, y) d_{x, y}^{*} G(x, y) .
$$

From Theorem 3.1 we can easily generalize it to the multi-parameter integral.

Definition 3.2 Let $E_{1} \times \cdots \times E_{n}=\left\{a_{1}=x_{1}^{0}<x_{1}^{1}<\cdots<x_{1}^{N_{1}}=\right.$ $\left.b_{1}, \cdots, a_{n}=x_{n}^{0}<x_{n}^{1}<\cdots<x_{n}^{N_{n}}=b_{n}\right\}$ be an arbitrary partition of $\left[a_{1}, b_{1}\right] \times \cdots\left[a_{n}, b_{n}\right]$. We call $F\left(x_{1}, \cdots, x_{n}\right)$ is of bounded $\Phi_{i}$-variation in $x_{i}$ uniformly in $\left(x_{1}, \cdots, x_{i-1}, x_{i+1}, \cdots, x_{n}\right), i=1, \cdots n$, if

$$
\sup _{x_{1}, \cdots, x_{i-1}, x_{i+1}, \cdots, x_{n}} \sup _{E_{i}} \sum_{k_{i}=1}^{N_{i}} \Phi_{i}\left(\left|\Delta_{x_{i}^{k_{i}-1}, x_{i}} F\right|\right)<\infty,
$$


Here $\Delta$ is the difference operator (see [1]) as follows,

$$
\begin{aligned}
\Delta_{x_{i}^{k_{i}-1}, x_{i}^{k_{i}}} F= & F\left(x_{1}, \cdots, x_{i-1}, x_{i}^{k_{i}}, x_{i+1}, \cdots, x_{n}\right) \\
& -F\left(x_{1}, \cdots, x_{i-1}, x_{i}^{k_{i}-1}, x_{i+1}, \cdots, x_{n}\right) .
\end{aligned}
$$

We call $G\left(x_{1}, \cdots, x_{n}\right)$ is of bounded $\Psi_{1}, \cdots, \Psi_{n}$-variation in $\left(x_{1}, \cdots, x_{n}\right)$, if

$\sup _{E_{1} \times \cdots \times E_{n}} \sum_{k_{n}=1}^{N_{n}} \Psi_{n}\left(\cdots\left(\sum_{k_{1}=1}^{N_{1}} \Psi_{1}\left(\left|\Delta_{x_{n}^{k_{n}-1}, x_{n}^{k_{n}}} \cdots \Delta_{x_{1}^{k_{1}-1}, x_{1}^{k_{1}}} G\right|\right)\right) \cdots\right)<\infty($

We say a function $f\left(x_{1}, \cdots, x_{n}\right)$ has a jump at $\left(x_{1}^{0}, \cdots, x_{n}^{0}\right)$ if there exists an $\varepsilon>0$ such that for any $\delta>0$, there exists $\left(x_{1}^{1}, \cdots, x_{n}^{1}\right)$ satisfying $\max \left\{\left|x_{1}^{0}-x_{1}^{1}\right|, \cdots,\left|x_{n}^{0}-x_{n}^{1}\right|\right\}<\delta$ and $\left|\Delta_{x_{n}^{0}, x_{n}^{1}}, \cdots, \Delta_{x_{1}^{0}, x_{1}^{1}} f\right|>$ $\varepsilon$. For a function $G\left(x_{1}, \cdots, x_{n}\right)$ of bounded $\Psi_{1}, \cdots, \Psi_{n}$-variation, for any given $\varepsilon>0$, it is easy to see that there exists a $\delta(\varepsilon)>0$ and a finite number of jump points $\left\{\left(x_{1}^{1}, \cdots, x_{n}^{1}\right), \cdots,\left(x_{1}^{m_{1}}, \cdots, x_{n}^{m_{n}}\right)\right\}$ such that $\left|\Delta_{\tilde{x}_{n}, x_{n}}, \cdots, \Delta_{\tilde{x}_{1}, x_{1}} G\right|<\varepsilon$ whenever $\max \left\{\left|\tilde{x}_{1}-x_{1}\right|, \cdots,\left|\tilde{x}_{n}-x_{n}\right|\right\}<\delta(\varepsilon)$, $\left[\tilde{x}_{i}, x_{i}\right] \cap\left\{x_{1}^{i}, \cdots, x_{n}^{m_{i}}\right\}=\emptyset$ for all $i=1,2, \cdots, n$. Denote $H_{10} \times \cdots \times H_{n 0}:=$ $\left\{x_{1}^{1}, \cdots, x_{1}^{m_{1}}\right\} \times \cdots \times\left\{x_{n}^{1}, \cdots, x_{n}^{m_{n}}\right\}$. In the following, we assume the following finite large jump condition: for any $\varepsilon>0$, there exists at most finite many points $\left\{x_{1}^{1}, \cdots, x_{1}^{m_{1}^{\prime}}\right\}, \cdots,\left\{x_{n}^{1}, \cdots, x_{n}^{m_{n}^{\prime}}\right\}$ and a constant $\delta(\varepsilon)>0$ such that for each $i=1,2, \cdots, n$, the total $\Psi_{1}, \cdots, \Psi_{n}$-variation of $G$ on $\left[x_{1}^{\prime}, x_{1}^{\prime \prime}\right] \times$ $\cdots\left[x_{i}, x_{i}+\delta\right] \times \cdots \times\left[x_{n}^{\prime}, x_{n}^{\prime \prime}\right]$ is smaller than $\varepsilon$ if $\left[x_{i}, x_{i}+\delta\right] \cap\left\{x_{i}^{1}, \cdots, x_{i}^{m_{i}^{\prime}}\right\}=\emptyset$. Denote $H_{1} \times \cdots \times H_{n}:=\left\{x_{1}^{1}, \cdots, x_{1}^{m_{1}^{\prime}}\right\} \times \cdots \times\left\{x_{n}^{1}, \cdots, x_{n}^{m_{n}^{\prime}}\right\}$. It is obvious that $H_{1} \times \cdots \times H_{n} \supset H_{10} \times \cdots \times H_{n 0}$.

Similarly we can define $m\left(E_{1} \times E_{2} \times \cdots \times E_{n}\right)$ as in Theorem 3.1 and get the theorem for multi-parameter integral.

Theorem 3.3 Let $F\left(x_{1}, \cdots, x_{n}\right)$ be a continuous function of bounded $\Phi_{i}$ variation in $x_{i}$ uniformly in $\left(x_{1}, \cdots, x_{i-1}, x_{i+1}, \cdots, x_{n}\right) ; i=1, \cdots n, G\left(x_{1}, \cdots, x_{n}\right)$ be of bounded $\Psi_{1}, \cdots, \Psi_{n}$-variation in $\left(x_{1}, \cdots, x_{n}\right)$ and satisfy the finite large jump condition, where $\Psi_{1}, \cdots, \Psi_{n}$ are convex functions. If there exist monotone increasing functions $\varrho_{1}, \cdots, \varrho_{n}$ subject to $\varrho_{1}(u) \cdots \varrho_{n}(u)=u$ such that

$$
\sum_{k_{n}=1}^{\infty} \cdots \sum_{k_{1}=1}^{\infty} \varrho_{1}\left[\varphi_{1}\left(\frac{1}{k_{1}}\right)\right] \cdots \varrho_{n}\left[\varphi_{n}\left(\frac{1}{k_{n}}\right)\right] \psi_{1}\left[\frac{1}{k_{1}}\left[\cdots \psi_{n}\left(\frac{1}{k_{n}}\right) \cdots\right]\right]<\infty
$$

then the integral

$$
\begin{aligned}
& \int_{a_{n}}^{b_{n}} \cdots \int_{a_{1}}^{b_{1}} F\left(x_{1}, \cdots, x_{n}\right) d_{x_{1}, \cdots, x_{n}} G\left(x_{1}, \cdots, x_{n}\right) \\
= & \lim _{m\left(E_{1} \times \cdots E_{n}\right) \rightarrow 0} \sum_{k_{n}=1}^{N_{n}} \cdots \sum_{k_{1}=1}^{N_{1}} F\left(x_{1}^{k_{1}-1}, \cdots, x_{n}^{k_{n}-1}\right)\left(\Delta_{x_{n}^{k_{n}-1}, x_{n}^{k_{n}}} \cdots \Delta_{x_{1}^{k_{1}-1}, x_{1}^{k_{1}}} G\right)
\end{aligned}
$$

is well defined, as long as $E_{1} \times E_{2} \times \cdots \times E_{n}$ include $H_{1} \times H_{2} \times \cdots \times H_{n}$. 


\section{Two-parameter integrals of local times}

Assume that $X=\left(X_{t}\right)_{t>0}$ is a continuous semi-martingale, $L_{t}^{x}$ is the local time of $X_{t}$ at $x$. By localization argument, we can assume that $X_{t}$ is bounded and its local time $L_{t}(x)$ is also bounded uniformly in $x$ (see [9]). We divide $[0, t] \times[-N, N]$ by $E \times E^{\prime}:=E_{[0, t]} \times E_{[-N, N]}^{\prime}=\left\{0=s_{0}<s_{1}<\cdots<s_{m}=\right.$ $\left.t,-N=x_{0}<x_{1}<\cdots<x_{l}=N\right\}$, where $[-N, N]$ covers the support of local time $L_{t}^{x}$.

In this section we will define $\int_{-\infty}^{\infty} \int_{0}^{t} g(s, x) d_{s, x} L_{s}^{x}$. We will first use Theorem 3.1 to define the integral $\int_{-\infty}^{\infty} \int_{0}^{t} \tilde{L}_{s}^{x} d_{s, x} g(s, x)$. Here $\tilde{L}_{s}^{x}$ refers to the continuous part in decompotition (2.13) of local times.

Theorem 4.1 Assume $g:[0, t] \times R \rightarrow R$ is of bounded $\Phi_{1}, \Psi_{1}$-variation in $(s, x)$, i.e. $\sup _{E \times E^{\prime}} \sum_{i=0}^{l-1} \Psi_{1}\left(\sum_{j=0}^{m-1} \Phi_{1}\left(\left|\Delta_{j} \Delta_{i} g\right|\right)\right)<\infty$ for the partition we defined as before and satisfy the finite large jump condition. Then if there exist monotone increasing functions $\varrho$ and $\sigma$ subject to $\varrho(u) \sigma(u)=u$ such that for $a \delta>0$

$$
\sum_{n, m} \varrho\left[\left(\frac{1}{n}\right)^{\frac{1}{2+\delta}}\right] \sigma\left(\frac{1}{m}\right) \varphi_{1}\left(\frac{1}{n} \psi_{1}\left(\frac{1}{m}\right)\right)<\infty
$$

the integral

$$
\begin{gathered}
\int_{-\infty}^{\infty} \int_{0}^{t} \tilde{L}_{s}^{x} d_{s, x} g(s, x) \\
=\lim _{m\left(E \times E^{\prime}\right) \rightarrow 0} \sum_{i=0}^{l-1} \sum_{j=0}^{m-1} \tilde{L}\left(s_{j}, x_{i}\right)\left(g\left(s_{j+1}, x_{i+1}\right)-g\left(s_{j+1}, x_{i}\right)\right. \\
\left.-g\left(s_{j}, x_{i+1}\right)+g\left(s_{j}, x_{i}\right)\right)
\end{gathered}
$$

is well defined for almost all $\omega \in \Omega$ in the sense of Theorem 3.1.

Proof: Note $\tilde{L}_{s}(x)$ is increasing in $s$ so of bounded variation in $s$. Let $h$ be defined by (2.14). It is easy to know from $(2.11), h(s, x)$ is of bounded variation in $s$. Therefore we have $\tilde{L}(s, x)$ is of bounded variation in $s$. In particular, using (2.13), (2.14) and (2.11) we obtain

$$
\sup _{E} \sum_{j=0}^{l-1}\left|\tilde{L}\left(s_{j+1}, x\right)-\tilde{L}\left(s_{j}, x\right)\right|=L_{t}(x)+\int_{0}^{t}\left|d V_{s}\right| \leq P
$$

where $P$ is a constant independent of $\mathrm{x}$. On the other hand, from Lemma 2.1 and Lemma 2.2, we know,

$$
\sup _{E^{\prime}} \sum_{i=0}^{m-1}\left|\tilde{L}\left(s, x_{i+1}\right)-\tilde{L}\left(s, x_{i}\right)\right|^{2+\delta} \leq Q
$$


where $Q$ is a constant independent of $s$. Therefore under condition (4.1), the integral $\int_{-\infty}^{\infty} \int_{0}^{t} \tilde{L}_{s}(x) d_{s, x} g(s, x)$ is well defined.

Corollary 4.1 Assume $g:[0, t] \times R \rightarrow R$ is of bounded $p, q$-variation, i.e. $\sup _{E \times E^{\prime}} \sum_{i=0}^{l-1}\left(\sum_{j=0}^{m-1}\left|\Delta_{j} \Delta_{i} g\right|^{p}\right)^{q}<\infty$, where $p, q \geq 1,2 q+1>2 p q$ and satisfy the finite large jump condition, then the integral

$$
\begin{aligned}
& \int_{-\infty}^{\infty} \int_{0}^{t} \tilde{L}_{s}^{x} d_{s, x} g(s, x) \\
= & \lim _{m\left(E \times E^{\prime}\right) \rightarrow 0} \sum_{i, j} \tilde{L}\left(s_{j}, x_{i}\right)\left(g\left(s_{j+1}, x_{i+1}\right)-g\left(s_{j+1}, x_{i}\right)\right. \\
& \left.-g\left(s_{j}, x_{i+1}\right)+g\left(s_{j}, x_{i}\right)\right)
\end{aligned}
$$

is well defined in the sense of Theorem 3.1.

Proof: For any $p, q \geq 1$ satisfying $2 q+1>2 p q$, we have $2\left(1-\frac{1}{p}\right)<\frac{1}{p q}$. Therefore there exists a number $\alpha$ such that $2\left(1-\frac{1}{p}\right)<\alpha<\frac{1}{p q}$. This implies that $\frac{\alpha}{2}+\frac{1}{p}>1$ and $1-\alpha+\frac{1}{p q}>1$. So there is a small $\delta>0$ such that $\frac{\alpha}{2+\delta}+\frac{1}{p}>1$ and $1-\alpha+\frac{1}{p q}>1$. Take $\varrho(u)=u^{\alpha}$ and $\sigma(u)=u^{1-\alpha}$, then it is easy to see that

$$
\sum_{n, m} \varrho\left[\left(\frac{1}{n}\right)^{\frac{1}{2+\delta}}\right] \sigma\left(\frac{1}{m}\right)\left(\frac{1}{n}\right)^{\frac{1}{p}}\left(\frac{1}{m}\right)^{\frac{1}{p q}}<\infty .
$$

Therefore the integral (4.3) is well defined.

After defining the integral $\int_{-\infty}^{\infty} \int_{0}^{t} \tilde{L}_{s}(x) d_{s, x} g(s, x)$, let's study the integral $\int_{-\infty}^{\infty} \int_{0}^{t} g(s, x) d_{s, x} \tilde{L}_{s}^{x}$. Note

$$
\begin{aligned}
& \sum_{i=0}^{l-1} \sum_{j=0}^{m-1} g\left(s_{j}, x_{i}\right)\left[\tilde{L}_{s_{j+1}}\left(x_{i+1}\right)-\tilde{L}_{s_{j}}\left(x_{i+1}\right)-\tilde{L}_{s_{j+1}}\left(x_{i}\right)+\tilde{L}_{s_{j}}\left(x_{i}\right)\right] \\
= & \sum_{i=1}^{l} \sum_{j=1}^{m} g\left(s_{j-1}, x_{i-1}\right) \tilde{L}_{s_{j}}\left(x_{i}\right)-\sum_{i=1}^{l} \sum_{j=0}^{m-1} g\left(s_{j}, x_{i-1}\right) \tilde{L}_{s_{j}}\left(x_{i}\right) \\
& -\sum_{i=0}^{l-1} \sum_{j=1}^{m} g\left(s_{j-1}, x_{i}\right) \tilde{L}_{s_{j}}\left(x_{i}\right)+\sum_{i=0}^{l-1} \sum_{j=0}^{m-1} g\left(s_{j}, x_{i}\right) \tilde{L}_{s_{j}}\left(x_{i}\right) \\
= & \sum_{i=1}^{l} \sum_{j=1}^{m} \tilde{L}_{s_{j}}\left(x_{i}\right)\left[g\left(s_{j}, x_{i}\right)-g\left(s_{j}, x_{i-1}\right)-g\left(s_{j-1}, x_{i}\right)+g\left(s_{j-1}, x_{i-1}\right)\right]
\end{aligned}
$$




$$
\begin{aligned}
& -\sum_{i=1}^{l}\left[g\left(0, x_{i-1}\right) \tilde{L}_{0}\left(x_{i}\right)-g\left(t, x_{i-1}\right) \tilde{L}_{t}\left(x_{i}\right)\right] \\
& -\sum_{j=1}^{m}\left[g\left(s_{j-1},-N\right) \tilde{L}_{s_{j}}(-N)-g\left(s_{j-1}, N\right) \tilde{L}_{s_{j}}(N)\right] \\
& +\sum_{j=0}^{m-1}\left[g\left(s_{j},-N\right) \tilde{L}_{s_{j}}\left(x_{0}\right)-g\left(s_{j}, N\right) \tilde{L}_{s_{j}}(N)\right] \\
& +\sum_{i=0}^{l-1}\left[g\left(0, x_{i}\right) \tilde{L}_{0}\left(x_{i}\right)-g\left(t, x_{i}\right) \tilde{L}_{t}\left(x_{i}\right)\right] \\
= & \sum_{i=1}^{l} \sum_{j=1}^{m} \tilde{L}_{s_{j}}\left(x_{i}\right)\left[g\left(s_{j}, x_{i}\right)-g\left(s_{j}, x_{i-1}\right)-g\left(s_{j-1}, x_{i}\right)+g\left(s_{j-1}, x_{i-1}\right)\right] \\
& -\sum_{i=1}^{l} \tilde{L}_{t}\left(x_{i}\right)\left(g\left(t, x_{i}\right)-g\left(t, x_{i-1}\right)\right) .
\end{aligned}
$$

Under the conditions of Theorem 4.1 and Proposition 3.1 and noticing that $\tilde{L}_{t}(x)$ is continuous in $t$, we know that the first term of (4.5) converges to $\int_{-\infty}^{\infty} \int_{0}^{t} \tilde{L}_{s}(x) d_{s, x} g(s, x)$, and from rough path integration of one parameter, we know that the second term converges to $\int_{-\infty}^{\infty} \tilde{L}_{t}(x) d_{x} g(t, x)$ if further $g(s, x)$ is of bounded $\gamma$-variation $(1 \leq \gamma<2)$ in $x$ uniformly in $s$. So the sum

$$
\sum_{i=0}^{l-1} \sum_{j=0}^{m-1} g\left(s_{j}, x_{i}\right)\left[\tilde{L}_{s_{j+1}}\left(x_{i+1}\right)-\tilde{L}_{s_{j}}\left(x_{i+1}\right)-\tilde{L}_{s_{j+1}}\left(x_{i}\right)+\tilde{L}_{s_{j}}\left(x_{i}\right)\right]
$$

converges, we denote its limit by

$$
\begin{gathered}
\int_{-\infty}^{\infty} \int_{0}^{t} g(s, x) d_{s, x} \tilde{L}_{s}^{x} \\
=\lim _{m\left(E \times E^{\prime}\right) \rightarrow 0} \sum_{i=0}^{l-1} \sum_{j=0}^{m-1} g\left(s_{j}, x_{i}\right)\left[\tilde{L}_{s_{j+1}}\left(x_{i+1}\right)-\tilde{L}_{s_{j}}\left(x_{i+1}\right)\right. \\
\left.-\tilde{L}_{s_{j+1}}\left(x_{i}\right)+\tilde{L}_{s_{j}}\left(x_{i}\right)\right]
\end{gathered}
$$

and

$$
\int_{-\infty}^{\infty} \int_{0}^{t} g(s, x) d_{s, x} \tilde{L}_{s}^{x}=\int_{-\infty}^{\infty} \int_{0}^{t} \tilde{L}_{s}^{x} d_{s, x} g(s, x)-\int_{-\infty}^{\infty} \tilde{L}_{t}(x) d_{x} g(t, x) .
$$

Now recall decomposition (2.13) and (2.14) and Lemma 2.2, as in Elworthy, Truman and Zhao [6], the integral $\int_{0}^{t} \int_{-\infty}^{\infty} g(s, x) d_{s, x} h(s, x)$ is defined as a two-parameter Lebesgue-Stieltjes integral. Therefore we can define 
$\int_{0}^{t} \int_{-\infty}^{\infty} g(s, x) d_{s, x} L(s, x)=\int_{0}^{t} \int_{-\infty}^{\infty} g(s, x) d_{s, x} \tilde{L}(s, x)+\int_{0}^{t} \int_{-\infty}^{\infty} g(s, x) d_{s, x} h(s, x)$.

Remark 4.1 If $g(s, x)$ is $C^{1}$ in $x$, we have

$$
\int_{-\infty}^{\infty} \int_{0}^{t} g(s, x) d_{s, x} L_{s}^{x}=-\int_{-\infty}^{\infty} \int_{0}^{t} \nabla g(s, x) d_{s} L_{s}(x) d x .
$$

This can be seen from the following. As one can always add some points in the partition to make $L_{s_{j}}^{x_{1}}=0$ and $L_{s_{j}}^{x_{l+1}}=0$ for all $j=1,2, \cdots, m$, as $L$ has a compact support in $x$, therefore

$$
\begin{aligned}
& \lim _{m\left(E \times E^{\prime}\right) \rightarrow 0} \sum_{i=1}^{l} \sum_{j=1}^{m} g\left(s_{j}, x_{i}\right)\left[L_{s_{j}}^{s_{j+1}}\left(x_{i+1}\right)-L_{s_{j}}^{s_{j+1}}\left(x_{i}\right)\right] \\
= & \lim _{m\left(E \times E^{\prime}\right) \rightarrow 0}\left(\sum_{i=1}^{l} \sum_{j=1}^{m} g\left(s_{j}, x_{i}\right) L_{s_{j}}^{s_{j+1}}\left(x_{i+1}\right)-\sum_{i=0}^{l-1} \sum_{j=1}^{m} g\left(s_{j}, x_{i+1}\right) L_{s_{j}}^{s_{j+1}}\left(x_{i+1}\right)\right) \\
= & -\lim _{m\left(E \times E^{\prime}\right) \rightarrow 0} \sum_{i=1}^{l} \sum_{j=1}^{m}\left[g\left(s_{j}, x_{i+1}\right)-g\left(s_{j}, x_{i}\right)\right] L_{s_{j}}^{s_{j+1}}\left(x_{i+1}\right) \\
= & -\lim _{m\left(E \times E^{\prime}\right) \rightarrow 0} \sum_{i=1}^{l} \sum_{j=1}^{m} \nabla g\left(s_{j}, \xi_{i}\right) L_{s_{j}}^{s_{j+1}}\left(x_{i+1}\right)\left(x_{i+1}-x_{i}\right) \\
= & -\lim _{m\left(E_{[-N, N]}^{\prime}\right) \rightarrow 0} \sum_{i} \int_{0}^{t} \nabla g\left(s, x_{i+1}\right) d_{s} L_{s}\left(x_{i+1}\right)\left(x_{i+1}-x_{i}\right) \\
& -\lim _{m\left(E_{[-N, N]}^{\prime}\right) \rightarrow 0} \sum_{i} \int_{0}^{t}\left(\nabla g\left(s, \xi_{i}\right)-\nabla g\left(s, x_{i+1}\right)\right) d_{s} L_{s}\left(x_{i+1}\right)\left(x_{i+1}-x_{i}\right) \\
= & -\int_{-\infty}^{\infty} \int_{0}^{t} \nabla g(s, x) d_{s} L_{s}(x) d x .
\end{aligned}
$$

Theorem 4.2 Let $f:[0, t] \times R \rightarrow R$ be of bounded $\gamma$-variation in $x$ uniformly in $s$ and of bounded $p, q$-variation in $(s, x)$ and satisfy the finite large jump condition, where $1 \leq \gamma<2$ and $p, q \geq 1,2 q+1>2 p q$, and

$$
f_{n}(s, x):=\int_{0}^{2} \int_{0}^{2} \rho(r) \rho(z) f\left(s-\frac{r}{n}, x-\frac{z}{n}\right) d r d z, \quad n \geq 1,
$$

where $\rho$ is the mollifier defined in (2.19). Then

$$
\int_{-\infty}^{\infty} \int_{0}^{t} f_{n}(s, x) d_{s, x} L_{s}^{x} \rightarrow \int_{-\infty}^{\infty} \int_{0}^{t} f(s, x) d_{s, x} L_{s}^{x}, \text { as } n \rightarrow \infty .
$$

Proof: First we can easily verify that $f_{n}$ are also of bounded $p, q$-variation. We extend $f$ to $s<0$ by defining $f(s, x) \equiv 0$, for $s<0$, and denote an arbitrary partition of $[0, t] \times[-N-2, N]$ by 
$E \times E_{1}^{\prime}:=\left\{0=s_{0}<s_{1}<\cdots<s_{m}=t,-N-2=x_{0}<x_{1}<\cdots<x_{l^{\prime}}=N\right\}$.

Because $[-N-2, N]$ also covers the compact support of local time, we have

$$
\sup _{E \times E_{1}^{\prime}} \sum_{i=1}^{l^{\prime}}\left(\sum_{j=1}^{m}\left|\Delta_{j} \Delta_{i} f\right|^{p}\right)^{q}=M,
$$

and

$$
\sup _{E_{1}^{\prime}} \sum_{i=1}^{l^{\prime}}\left|f\left(s, x_{i}\right)-f\left(s, x_{i-1}\right)\right|^{\gamma}=M^{\prime}
$$

where $M$ and $M^{\prime}$ are constants. So by Hölder inequality,

$$
\begin{aligned}
& \sum_{i=1}^{l}\left(\sum_{j=1}^{m}\left|\Delta_{j} \Delta_{i} f_{n}\right|^{p}\right)^{q} \\
= & \sum_{i=1}^{l}\left(\sum_{j=1}^{m}\left|\int_{0}^{2} \int_{0}^{2} \rho(r) \rho(z) \Delta_{j} \Delta_{i} f\left(\cdot-\frac{r}{n}, \cdot-\frac{z}{n}\right) d r d z\right|^{p}\right)^{q} \\
\leq & A \sum_{i=1}^{l}\left(\int_{0}^{2} \int_{0}^{2} \sum_{j=1}^{m}\left|\Delta_{j} \Delta_{i} f\left(\cdot-\frac{r}{n}, \cdot-\frac{z}{n}\right)\right|^{p} d r d z\right)^{q} \\
\leq & B \int_{0}^{2} \int_{0}^{2} \sum_{i=1}^{l}\left(\sum_{j=1}^{m}\left|\Delta_{j} \Delta_{i} f\left(\cdot-\frac{r}{n}, \cdot-\frac{z}{n}\right)\right|^{p}\right)^{q} d z d r \\
\leq & B \int_{0}^{2} \int_{0}^{2} \sup _{E \times E_{1}^{\prime}} \sum_{i=1}^{l^{\prime}}\left(\sum_{j=1}^{m}\left|\Delta_{j} \Delta_{i} f\right|^{p}\right)^{q} d r d z \\
\leq & M_{1},
\end{aligned}
$$

where $A, B$ and $M_{1}$ (independent of $n$ ) are constants. Also from the above estimate, the finite large jump condition for $f_{n}$ when $n$ is sufficiently large follows from the finite large jump assumption of $f$. Similarly,

$$
\begin{aligned}
& \sum_{i=1}^{l}\left|f_{n}\left(s, x_{i}\right)-f_{n}\left(s, x_{i-1}\right)\right|^{\gamma} \\
= & \sum_{i=1}^{l} \mid \int_{0}^{2} \int_{0}^{2} \rho(r) \rho(z)\left(f\left(s-\frac{r}{n}, x_{i}-\frac{z}{n}\right)-\left.f\left(s-\frac{r}{n}, x_{i-1}-\frac{z}{n}\right) d r d z\right|^{\gamma}\right. \\
\leq & C \int_{0}^{2} \int_{0}^{2} \sum_{i=1}^{l}\left|f\left(s-\frac{r}{n}, x_{i}-\frac{z}{n}\right)-f\left(s-\frac{r}{n}, x_{i-1}-\frac{z}{n}\right)\right|^{\gamma} d r d z
\end{aligned}
$$




$$
\begin{aligned}
& \leq C \int_{0}^{2} \int_{0}^{2} \sup _{E^{\prime}} \sum_{i=1}^{l^{\prime}}\left|f\left(s, x_{i}\right)-f\left(s, x_{i-1}\right)\right|^{\gamma} d r d z \\
& \leq M_{2}
\end{aligned}
$$

where $C$ and $M_{2}$ (independent of $n$ ) are constants. So the integral $\int_{-\infty}^{\infty} \int_{0}^{t} f_{n}(s, x) d_{s, x} L_{s}^{x}$ is well defined, by argument we discussed before,

$$
\begin{aligned}
& \int_{-\infty}^{\infty} \int_{0}^{t} f_{n}(s, x) d_{s, x} L_{s}^{x} \\
= & \int_{-\infty}^{\infty} \int_{0}^{t} \tilde{L}_{s}^{x} d_{s, x} f_{n}(s, x)-\int_{-\infty}^{\infty} \tilde{L}_{t}^{x} d_{x} f_{n}(t, x) \\
& +\int_{-\infty}^{\infty} \int_{0}^{t} f_{n}(s, x) d_{s, x} h(s, x) .
\end{aligned}
$$

For such $p, q$ satisfying $p, q \geq 1$, and $2 q(p-1)<1$, there exist a small positive number $\delta>0$ such that $(2+\delta) q(p+\delta-1)<1$, so

$$
\sum_{n, m} \varrho\left[\left(\frac{1}{n}\right)^{\frac{1}{2+\delta}}\right] \sigma\left(\frac{1}{m}\right)\left(\frac{1}{n}\right)^{\frac{1}{p+\delta}}\left(\frac{1}{m}\right)^{\frac{1}{(p+\delta) q}}<\infty
$$

still holds for $\rho(u)=u^{\alpha}, \sigma(u)=u^{1-\alpha}$, where $(2+\delta)\left(1-\frac{1}{p+\delta}\right)<\alpha<\frac{1}{(p+\delta) q}$. By Theorem 3.2 and Proposition 3.1, we can pass the limit to get

$$
\lim _{n \rightarrow \infty} \int_{-\infty}^{\infty} \int_{0}^{t} \tilde{L}_{s}^{x} d_{s, x} f_{n}(s, x)=\int_{-\infty}^{\infty} \int_{0}^{t} \tilde{L}_{s}^{x} d_{s, x} f(s, x) .
$$

Using a similar method as in the proof of Theorem 2.1, we can prove that

$$
\lim _{n \rightarrow \infty} \int_{-\infty}^{\infty} \tilde{L}_{t}^{x} d_{x} f_{n}(t, x)=\int_{-\infty}^{\infty} \tilde{L}_{t}^{x} d_{x} f(t, x) .
$$

The convergence of the last term $\int_{-\infty}^{\infty} \int_{0}^{t} f_{n}(s, x) d_{s, x} h(s, x)$ in (4.9) follows from Lebesgue's dominated convergence theorem. So we proved the desired result.

Theorem 4.3 Let $X=\left(X_{s}\right)_{s \geq 0}$ be a continuous semi-martingale and assume $f:[0, \infty) \times R \rightarrow R$ satisfies

(i) $f$ is left continuous and locally bounded, with $f(t, x)$ jointly continuous from the right in $t$ and left in $x$ at each point $(0, x)$,

(ii) the left derivatives $\frac{\partial^{-}}{\partial t} f$ and $\nabla^{-} f$ exist at all points of $(0, \infty) \times R$ and $[0, \infty) \times R$ respectively,

(iii) $\frac{\partial^{-}}{\partial t} f$ and $\nabla^{-} f$ are left continuous and locally bounded,

(iv) $\nabla^{-} f(t, x)$ is of bounded $\gamma$-variation in $x$ uniformly in $t$ and of bounded $p, q$-variation in $(t, x)$ and satisfies the finite large jump condition, where $1 \leq \gamma<2$, and $p, q \geq 1,2 q+1>2 p q$. 
Then we have:

$$
\begin{aligned}
f\left(t, X_{t}\right)= & f\left(0, X_{0}\right)+\int_{0}^{t} \frac{\partial^{-}}{\partial s} f\left(s, X_{s}\right) d s+\int_{0}^{t} \nabla^{-} f\left(s, X_{s}\right) d X_{s} \\
& -\int_{0}^{t} \int_{-\infty}^{\infty} \nabla^{-} f(s, x) d_{s, x} L_{s}^{x}
\end{aligned}
$$

where $L_{t}^{x}$ is the local time of $X_{t}$ at $x$, the last integral is defined in (4.6).

Proof: Similar to the proof in [6], we can use smoothing procedure and take the limit to prove our result. The main different key point is the following : by Remark 4.1 and Theorem 4.2 ,

$$
\begin{aligned}
& \frac{1}{2} \int_{0}^{t} \Delta f_{n}\left(s, X_{s}\right) d<M>_{s} \\
= & \int_{-\infty}^{\infty} \int_{0}^{t} \Delta f_{n}(s, x) d L_{s}^{x} d x \\
= & -\int_{-\infty}^{\infty} \int_{0}^{t} \nabla f_{n}(s, x) d_{s, x} L_{s}^{x} \\
\rightarrow & -\int_{-\infty}^{\infty} \int_{0}^{t} \nabla^{-} f(s, x) d_{s, x} L_{s}^{x},
\end{aligned}
$$

when $n \rightarrow \infty$.

Example 4.1 Consider a function $f(t, x)=x^{3} t^{3} \cos \left(\frac{1}{t}+\frac{1}{x}\right)$ for $t, x \neq 0$ and $f(t, 0)=f(0, x)=f(0,0)=0$. This function is $C^{1,1}$ and its derivative about $x$ is $\frac{\partial}{\partial x} f(t, x)=3 t^{3} x^{2} \cos \left(\frac{1}{t}+\frac{1}{x}\right)+x t^{3} \sin \left(\frac{1}{t}+\frac{1}{x}\right)$ for $t, x \neq 0$ and $\frac{\partial}{\partial x} f(t, 0)=\frac{\partial}{\partial x} f(0, x)=\frac{\partial}{\partial x} f(0,0)=0$. It is easy to see that $\frac{\partial}{\partial x} f(t, x)$ is of unbounded variation in $x$ and in $(t, x)$, but of $\gamma$-variation in $x$ for any $\gamma>1$, $p, 1$-variation in $(t, x)$ for any $p>1$ (similar to Example 3.1). So Theorem 4.3 can be used.

Finally we would like to mention that our result should also work for stable processes noticing the $p$-variation result on the local times of stable processes studied by Marcus and Rosen [21]. But we should also point out that Marcus and Rosen's definition to $p$-variation is different from ours. But we can extend the proof of Lemma 2.1 to stable process. For the length of the paper, these results are not included in this paper.

\section{Acknowledgement}

We would like to thank N. Eisenbaum, K.D. Elworthy, T. Lyons, Z.M. Ma, Z. Qian, T. Zhang and S. Peng for useful conversation, especially to T. Lyons who introduced the idea of Young integral to us during the UK-Japan Winter School in January 2003 at Warwick University and to S. Peng for his hospitality and useful conversations during HZ's visit to Shandong University where the paper was finalized. EPSRC's grant no. GR/R69518 is gratefully acknowledged. 


\section{References}

1. R. B. Ash and C. A. Doláns-Dade. Probability and Measure Theory, Second Edition, Academic Press, (2000).

2. J. Azéma, T. Jeulin, F. Knight and M. Yor, Quelques calculs de compensateurs impliquant l'injectivité de certauns processus croissants, Séminaire de Probabilités XXXII (1998), LNM1686, 316-327.

3. R. F. Bass, B. M. Hambly and T. J. Lyons (1998), Extending the Wong-Zakai theorem to reversible Markov processes, J. Euro. Math. Soc., 4(2002), 237-269.

4. N. Bouleau and M. Yor, Sur la variation quadratique des temps locaux de certaines semimartingales, C.R.Acad, Sci. Paris, Ser.I Math 292 (1981), 491494.

5. G. A. Brosamler, Quadratic variation of potentials and harmonic functions, Transactions of the American Mathematical Society 149, 243-257, 1970.

6. K. D. Elworthy, A. Truman and H. Z. Zhao, Generalized Itô Formulae and space-time Lebesgue-Stieltjes integrals of local times, Séminaire de Probabilités, vol 40 (to appear).

7. N. Eisenbaum, Integration with respect to local time, Potential analysis 13 (2000), 303-328.

8. N. Eisenbaum, Local time-space calculus for revisible semi-martingales, Séminaire de Probabilités vol 40, Lectures Notes in Mathematics, SpringerVerlag (to appear).

9. C. R. Feng and H. Z. Zhao, A Generalized Itô's Formula in Two-Dimensions and Stochastic Lebesgue-Stieltjes Integrals, preprint 2004.

10. F. Flandoli, F. Russo and J. Wolf, Some stochastic differential equations with distributional drift, Osaka J. Math. 40 (2003), no. 2, 493-542.

11. H. Föllmer and P. Protter, On Itô Formula for multidimensional Brownian motion, Probability Theory and Related Fields 116 (2000), 1-20.

12. R. Ghomrasni and G. Peskir, Local time-space caculus and Extensions of Itô's Formula (2003).

13. B. M. Hambly any T. L. Lyons (1998), Stochastic area for Brownian motion the Sierpinski gasket, Ann. Prob., 26, 132-48.

14. I. Karatzas and S. E. Shreve, Brownian Motion and Stochastic Calculus, Second Edition, preprint (Springer-Verlag: New York, 1998).

15. W. Kendall, The radial part of a $\Gamma$-martingale and non-implosion theorem, The Annals of Probability, 23, 479-500, 1995.

16. M. Ledoux, T. J. Lyons and Z. Qian, Lévy area of Wiener processes in Banach Spaces, Ann. Prob., Vol. 30, 546-578, 2002.

17. P. Lévy Processus Stochastiques et Mouvement Brownien, Gauthier-Villars, Paris, (1948).

18. T. Lyons Differential equations driven by rough signals (I): An extension of an inequality of L. C. Young. Math. Res. Lett., 1, 451-64, 1994.

19. T. Lyons Differential equations driven by rough signals Rev. Mat. Iberoamer., 14, 215-310, 1998.

20. T. Lyons and Z. Qian, System Control and Rough Paths, Clarendon Press Oxford, 2002.

21. M. B. Marcus and J. Rosen, p-variation of the local times of symmetric stable processes and of Gaussian processes with stationary increments, Ann. Prob. Vol.20 (1992), 1685-1713. 
22. P. A. Meyer, Un cours sur les intégrales stochastiques, Sém. Probab 10, Lecture Notes in Math, No. 511, Springer-velay (1976), 245-400.

23. S. Moret and D. Nualart, Generalization of It's formula for smooth nondegenerate martingales, Stochastic Process. Appl. 91, 115-149, 2001.

24. G. Peskir, A change-of-variable formula with local time on curves, J.Theoret Probab. (to appear).

25. G. Peskir, A change-of-variable formula with local time on surfaces, Séminaire de Probabilités vol 40, Lectures Notes in Mathematics, Springer-Verlag (to appear).

26. D. Revuz and M. Yor, Continuous Martingales and Brownian Motion, Second Edition, (Springer-Verlag: Berlin, Heidelberg, 1994).

27. L. C. G. Rogers and J. B. Walsh, Local time and stochastic area integrals, Annals of Probas. 19(2) (1991), 457-482.

28. H. Tanaka, Note on continuous additive functionals of the 1-dimensional Brownian path, Z.Wahrscheinlichkeitstheorie and Verw Gebiete 1 (1963), 251257.

29. J. B. Walsh, An Introduction to Stochastic Partial Diffenrential Equations, In École dété de Probabilité de Saint Flour, XIV, ed. D.L. Hennequin, Lecture Notes in Mathematics No. 1180, (1986), 265-439.

30. L. C. Young, An inequality of Hölder type, connected with Stieltjes integration, Acta Math., 67 (1936), 251-282.

31. L.C. Young, General inequalities of Stieltjes integrals and the convergence of Fourier series, Math. Ann., 115(1938), 581-612. 